\title{
LE ROLE DE L'AUREOMYGINE \\ DANS LA CROISSANCE PONDÉRALE DU RAT BLANC *
}

\author{
PAR
}

Claude CALET avec la collaboration d'Alain RERAT

\section{PLAN DU MÉMOIRE}

\section{INTRODUCTION}

Historique bref.

Rappel des hypothèses émises sur le mode d'action des antibiotiques comme facteur de croissance.

Orientation des recherches entreprises.

\section{CHAPITRE; PREMIER}

\section{EFFET D'ÉPARGNE DES ANTIBIOTIQUES POUR LES VITAMLNES} DU GROUPE B

I. - Influence des antibiotiques sur la réserve vitaminique du foie.
A. - Position du problème.
B. - Conditions expérimentales.
C. - Résultats expérimentaux.
a) Observations sur animal.
b) Réserves vitaminiques.
D. - Discussion.

II. - Mise en évidence du pouvoir d'épargne.

Io Conditions expérimentales et observations in vivo.

$2^{\circ}$ Etude biochimique des foies.

\section{Conclusion.}

* Ce travail a été effectué au Laboratoire de B iochimie de la Nutrition du Centre national de la Recherche Scientifique à Bellevue par Claude CALET, chargé de Recherches, et Alain RERAT, assistant, à l'Institut National de la Recherche agronomique, avec la collaboration technique de G. BALIVET.

C'est avec une grande tristesse et une profonde reconnaissance que je me rappelle Gérard BALIVET qui a trouvé la mort tragiquement dans un accident routier. BALIVET fut pour moi plus qu'un collaborateur technique mais un véritable ami. Il fut le compagnon de tous les instants dont les qualités et le dévouement sans limite ont permis de mener à bien ce travail.

C. C. 


\section{CHAPITRE, II}

\section{INFLUENCE DES ANTIBIOTIQUES SUR LA SYNTHËSE MICROBIENNE DES VITAMINES B}

I. - Première expérience.

a) Conditions expérimentales.

b) Résultats expérimentaux.

II. - Deuxième expérience.

a) Conditions expérimentales.

b) Résultats expérimentaux.

Io Résultats totaux.

$2^{\circ}$ Répartition des différentes formes vitaminiques synthétisées.

$3^{\circ}$ Rapport entre les vitamines synthétisées et celles retenues dans le foie.

c) Observations et mesures sur animaux.

Conclusion.

CHAPITRE III

\section{SIGNIFICATION DES RELATIONS ENTRE VITAMINE B12 ET AUREOMYCINE}

I. - Première expérience : Interchangeabilité vitamine B12-Auréomycine.

A. - Conditions expérimentales.

B. - Résultats expérimentaux.

a) Aspect des animaux.

b) Croissance et indice de consommation.

c) Êtude de la teneur vitaminique des foies.

Conclusion.

II. - Deuxième expérience : Rôle de la vitamine B12 comme facteur de croissance.

A. - Conditions expérimentales.

B. - Résultats expérimentaux (croissance et teneur hépatique en $\mathrm{BI} 2$ ).

III. - Conclusion générale.

\section{CHAPITRE IV}

SIGNIFICATION DE L'INFLUENCE DE L'A UR ÉOMYCINE SUR LA CROISSANCE D'APRÈS L'ÉTUDE DES BILANS
A. - Conditions expérimentales.
B. - Résultats expérimentaux.
- Teneur en eau des organes.
- Digestibilité.
- Rétention azotée.
C. - Discussion.
D. - Conclusions. 


\section{CHAPITRE V}

\section{SIGNIFICATION DE L'INFL UENCE DE L'A UREOMYCINE SUR LA CROISSANCE D'APRES L'ANALYSE DES TISSUS}

I. - Lipogénèse totale.

A. - Conditions expérimentales.

B. - Résultats expérimentaux.

a) Aspect des animaux.

b) Croissance.

c) Analyse corporelle des rats. Io Séries expérimentales et séries de référence. $2^{\circ}$ Rôle de l'auréomycine.

$3^{\circ}$ Cas particulier des cendres.

$4^{\circ}$ Rôle de la choline.

$5^{\circ}$ Discussion.

II. - Cas des acides nucléiques.

III. - Lipogénèse localisée.

IV. - Lipogénèse hépatique.

A. - Conditions expérimentales.

B. - Résultats expérimentaux.

a) État général et croissance.

b) Analyse des foies.

C. - Discussion des résultats.

a) Azote total.

b) Lipides.

D. - Conclusions.

\section{LE ROLE DES ANTIBIOTIQUES COMME FACTEURS DE SENESCENCE}

1. - Rendement énergétique de la ration.

II. - Facteur de sénescence.

\section{RÉSUMÉ ET CONCLUSIONS GENERALES}

\section{TECHNIQUES GÉNÉRALES}

\section{BIBLIOGRAPHIE}

Que M. le Professeur Jacquot, Directeur du Laboratoire de la Nutrition du C. N. R. S. à Bellevue trouve ici l'expression de ma vive reconnaissance pour la bienveillance avec laquelle il m'a pris comme élève et pour l'aide intellectuelle et morale qu'il n'a jamais cessé de m'apporter. 


\section{INTRODUCTION}

PAR

\section{CALET}

\section{I. - L'EFFET DES ANTIBIOTIQUES EN ALIMENTATION ANIMALE}

Il est bien connu que les protéines d'origine animale ont une efficacité supérieure à celle des protéines d'origine végétale. La cause en est, avant tout, dans le meilleur équilibre des aminoacides, équilibre qui se traduit in vivo par la Valeur Biologique. Un autre élément de supériorité a été découvert il y a quelques années et décrit sous le nom de "Facteur des Protéines Animales" ou A.P.F. (Animal Protein Factor) (II).

L'usage des antibiotiques en alimentation animale est lié à l'histoire de 1'A.P.F.

Au début, on extrayait ce facteur soit du foie (extraits hépatiques) $(87-205-288-289)$, soit du poisson (solubles de poisson; fish solubles) $(230-320)$, soit des excréments de ruminants $(\mathrm{r} 98-360-36 \mathrm{I})$ et de poules $(180-363)$. Ajoutés à un régime végétal, ces extraits augmentaient l'efficacité protidique de la ration sans qu'il y ait supplémentation par les acides aminés au sens classique du terme (II2-344). L'A. P. F. était une sorte de vitamine de croissance apte à valoriser les protides d'origine végétale. On ignorait encore sa nature exacte, mais on savait déjà que la vitamine BI2 était un de ses constituants essentiels $(269-328)$.

Par la suite, les sous-produits de l'industrie des antibiotiques furent utilisés en alimentation animale comme source de vitamine $\mathrm{BI} 2(420-$ $422)$. Or, on s'aperçut bien vite que ces sous-produits agissaient favorablement sur la croissance et l'indice de consommation (1'indice de consommation est d'après FÉVRIER (I60) la quantité de nourriture sèche ingérée par unité de gain de poids). En analysant les résultats et par recoupement, plusieurs auteurs montrèrent que l'efficacité de ces sousproduits dépassait de loin celle de la vitamine BI2 utilisée seule à l'état cristallisé ( $140-\mathrm{I} 78-26 \mathrm{I}-39 \mathrm{I})$. Quel était donc l'indéterminé responsable en grande partie d'une telle supériorité? On le sut bientôt : c'était les antibiotiques contenus dans les sous-produits industriels.

On a pu, en effet, démontrer directement que l'auréomycine cristallisée (4I7-4I9) augmentait la vitesse de croissance du Porc et améliorait 1'indice de consommation du régime (I60). Le mérite d'une telle démonstration, grosse de conséquences pratiques, revient à S'TOckSTAD et JUKES en I950 (240). Notons, cependant, qu'en I946 MOORE (308) avait déjà fait allusion à l'effet de croissance de la streptomycine. Les résultats de STOCKSTAD et JukEs furent rapidement confirmés et étendus à d'autres 
espèces animales (CARPENTER (83) Luecke (28o) sur Porc, Whitehili (455) sur Poussin, Stockstad (4I6) sur Dindon).

Si les travaux que nous venons de résumer le plus succinctement possible ont eu le mérite d'établir le rôle nutritionnel des antibiotiques, ils sont, par contre, responsables de deux erreurs. D'une part, on commercialise aujourd'hui sous le nom d'A.P.F. les sous-produits de l'industrie des antibiotiques qui sont essentiellement d'origine végétale. D'autre part, on diffuse largement dans le public ces spécialités dont on ignore encore le mécanisme d'action.

On sait aujourd'hui que l'effet des antibiotiques dépend de nombreux facteurs que nous passerons en revue.

Cette étude a été grandement facilitée grâce à l'excellente mise au point de Vachei, et F́́.

\section{A. - Nature et doses des antibiotiques.}

L'effet nutritionnel des antibiotiques est très variable. Ainsi dans le cas des volailles, pour un régime et une dose donnés, le bénéfice pondéral qu'on peut en attendre varie de o à $37 \mathrm{p}$. Ioo (33I - 383).

Certains antibiotiques n'ont aucune efficacité pour la croissance et ne sont pas utilisés en pratique zootechnique. Tel est le cas pour la chloromycétine (3I - 85-439-449), la néomycine (3I - 28I), la polymixine (II9), la subtiline (II9), la nysine (I7) et la rimocidine (282 - 377).

D'autres sont couramment employés par les éleveurs sans qu'il y ait de relation entre le domaine d'action bactériostatique et l'effet de croissance. En France, on utilise notamment :

- l'auréomycine à la dose de 9 à $20 \mathrm{mg} / \mathrm{kg}$ de ration (326)

- la pénicilline à la dose de I à $20 \mathrm{mg} / \mathrm{kg}$ de ration (2I7-29I)

- la bacitracine à la dose de 5 à $\mathrm{I} 5 \mathrm{mg} / \mathrm{kg}$ de ration (326)

- la streptomycine à la dose de 20 à $50 \mathrm{mg} / \mathrm{kg}$ de ration (76)

- la terramycine à la dose de ro à $20 \mathrm{mg} / \mathrm{kg}$ de ration (217-359).

La dose optima varie pour chaque espèce. En deçà, la réponse est proportionnelle à la quantité d'antibiotique; elle diminue sila dose allouée diminue. Au delà, une surcharge n'a pas d'effet supplémentaire (15-23$\left.55-7^{2}-83-85-94-125-267-28 \mathrm{I}-336-425\right)$.

\section{B. - Composition du régime.}

L'effet nutritionnel des antibiotiques est en grande partie fonction du régime utilisé. Il n'est pas possible d'établir une hiérarchie absolue des antibiotiques, mais uniquement un classement selon les types de ration. Voici quelques exemples : chez le Porc, avec une ration à base d'arachide, 
l'auréomycine surclasse les autres antibiotiques et la pénicilline vient en dernier (I25). A l'opposé, la pénicilline prend la première place avec une ration à base de soja et l'auréomycine se montre beaucoup moins efficace (455). (Ces faits ont été confirmés (64-76)).

Chez le Poulet, un régime contenant de la farine de poisson est valorisé par l'auréomycine, alors qu'il est déprécié par la pénicilline (6o). Ce résultat est d'ailleurs contesté (40).

Pour certains (64), la vitamine BI2 serait nécessaire pour obtenir le plein effet de l'antibiotique. Pour d'autres (9o), au contraire, la présence ou l'absence de BI2 n'influence pas le résultat. Ces faits montrent toute la complexité du rôle des antibiotiques et indiquent déjà que celui-ci est contingent de multiples facteurs alimentaires.

Néanmoins, deux notions peuvent déjà être assurées :

$\mathrm{I}^{0}$ en valeur absolue et dans la pratique, les meilleurs résultats sont obtenus avec une ration équilibrée enrichie en antibiotiques $(44-327)$;

$2^{\circ}$ en valeur relative, le bénéfice pondéral imputable aux aritibiotiques est d'autant plus important que le régime de base est lui-même plus déséquilibré (29I). Il y aura plus d'écart entre les témoins et les animaux traités dans le cas d'un régime déficient en vitamines $B$ ou en acides aminés indispensables que dans le cas d'un régime complet équilibré $(76-\mathrm{r} 22-\mathrm{I} 23-2 \mathrm{r} 3-240-29 \mathrm{r}-450)$. Ceci est en accord avec le pouvoir d'épargne que les antibiotiques ont vis-à-vis de divers éléments nutritionnels.

\section{C. - Age et espèce animale.}

Il est bien établi que les antibiotiques ont d'autant plus d'effet que l'animal est plus jeune $(54-222-323-438)$.

Ceci vient d'être particulièrement bien démontré par Fí́VRIER chez le porcelet à la mamelle (369). Toutefois, les divers antibiotiques n'ont pas la même action en fonction de l'âge : la pénicilline donne le meilleur résultat chez le Poulet de 4 semaines, mais à 8 semaines, c'est le cas de la terramycine (58).

Les antibiotiques sont surtout employés pour le Porc et certains types de volailles - en particulier le Poussin (218-324-326) et le Dindon ( $13-372-386-4 \mathrm{I} 6$ ). Pour l'Oie, on recommande la pénicilline (40I), la terramycine et l'auréomycine (59). Pour le Canard, les antibiotiques ont peu (2I6) ou pas d'effets (57).

Chez la Souris, les résultats peuvent être spectaculaires mais dépendent beaucoup du type de ration (437).

Chez le Chien, on note une légère action de l'auréomycine (9).

Chez le Lapin, aucun effet n'est enregistré $(225-424)$.

Chez les bovins et ovins, on obtient en général des réponses de 20 à 30 p. I00, chez les jeunes qui ne ruminent pas encore et qui se compor- 
tent physiologiquement comme des monogastriques $(26$ - III -247 $-313-365)$. Chez les ruminants adultes, les antibiotiques n'ont pas d'effets $\left(28-\mathrm{x}_{7} 6-229-237-3 \mathrm{r} 9\right)$ ou même sont nocifs en inhibant la flore du Rumen et en abaissant la digestibilité de la cellulose ( 36 - IOI - I70 - 202 - 245).

Enfin chez l'Homme, plusieurs auteurs ont cité l'action des antibiotiques pour compenser le retard des prématurés (133-357-403).

Par ailleurs, il semble exister une sorte d'accoutumance aux antibiotiques qui se traduit par une diminution d'efficacité, si on en prolonge l'usage. Cette accoutumance diffère du facteur âge, car elle se manifeste sur l'élevage entier. C'est ce qui ressort notamment des essais récents de WAIBEL et col. (442). Ces auteurs étudient l'influence de l'auréomycine et de la pénicilline sur la croissance du Poulet de batterie. Pendant les premiers 22 mois, les effets sont positifs dans l'ensemble ( $90 \mathrm{p}$. Ioo des cas) et importants (augmentation pondérale pouvant aller jusqu'à 39 p. Ioo). A partir $\mathrm{du}_{22^{\mathrm{e}}}$ mois, les réponses s'estompent et seule la moitié des lots mis en expérience réagit très légèrement (augmentation pondérale ne dépassant pas Io p. Ioo). La perte d'efficacité se traduit donc sur le double plan du nombre et de l'intensité des réponses favorables. Si ces résultats sont vérifiés, ils limitent singulièrement l'intérêt futur des antibiotiques en alimentation animale.

\section{D. - Etat sanitaire des sujets.}

Les résultats les plus spectaculaires (plus de roo p. roo d'augmentation de gain de poids) ont été obtenus chez les tout jeunes porcs (3I 8 $322-46 \mathrm{I})$ et les veaux de lait $(274-275-338-364)$, animaux particulièrement prédisposés aux troubles digestifs. Ainsi beaucoup d'auteurs pensent quel'effet des antibiotiques sur le développement ne porte pas directement sur la croissance, mais relève d'une action secondaire, consécutiveà la guérison des diarrhées.

Ces résultats ont été contestés par plusieurs auteurs pour lesquels la pénicilline ne diminue pas la fréquence des diarrhées $(5$ I -248$)$.

\section{E. - Autres efiets des antibiotiques.}

En plus de leur influence sur la croissance, les antibiotiques ont d'autres répercussions dont l'éleveur peut tirer parti.

Ils améliorent en général l'indice de consommation et par là-même le rendement alimentaire des rations $\left(35^{6}\right)$.

Ils stimulent l'appétence et nous verrons la signification qu'attribue FÉVRIER à ce facteur.

Par voie de conséquence, ils augmentent la soif des animaux, ce qui a 
laissé croire à une plus forte rétention d'eau et à une grande hydratation de la carcasse $(90-452)$, faits contestés comme nous le verrons $(62-$ 356).

Ils améliorent l'aspect général en donnant du lustre et de la qualité au poil ou à la plume.

Ils conduisent à une meilleure homogénéité des lots (89), du fait que les culots de portée et les sujets chétifs en retirent plus de bénéfice que les sujets vigoureux. Du point de vue de la sélection, c'est du reste une faute de maintenir en vie et de " doper " des animaux génétiquement déficients. D'où l'usage qui prévaut en France et dans de nombreux pays d'interdire les antibiotiques dans la ration des reproducteurs.

Cette brève revue indique déjà que l'efficacité des antibiotiques est conditionnée par de très nombreux facteurs. Elle montre, surtout, que même dans le domaine des applications pratiques, rien n'est sûr. Des faits tenus pour établis sont remis en question. Des résultats positifs sont suivis d'observations contraires. Jusqu'ici, l'emploi alimentaire des antibiotiques relève donc du pur empirisme. Ce ne sont malheureusement pas les investigations scientifiques (assez rares par rapport aux essais zootechniques) qui aident à la solution du problème, car il n'est pas exagéré de dire que l'obscurité la plus grande entoure encore le mode d'action des antibiotiques.

\section{II. - MODE D'ACTION DES ANTIBIOTIQUES}

Nous allons exposer successivement les hypothèses formulées pour expliciter le mécanisme selon lequel agissent les antibiotiques.

$1^{0}$ On a tenté de relier l'efficacité nutritionnelle des antibiotiques à leurs propriétés bactériostatiques plus ou moins étendues. Les faits suivants plaident en faveur de ce rapprochement :

- les antibiotiques ne forment pas une famille chimique définie, mais groupent des substances les plus variées dont la seule propriété commune est le caractère bactériostatique ;

- les médicaments bactéricides qui ne sont pas des antibiotiques, c'est-à-dire qui ne sont pas élaborés par des microorganismes, ont également un certain effet sur le développement pondéral ; c'est le cas pour les sulfamides (I $97-308-380-43 \mathrm{I})$;

- les antibiotiques sont inefficaces pour les animaux à tube digestif stérile (germ free) (276), ainsi que sur le développement de l'embryon de poulet qui est nécessairement stérile (238) ;

- ils sont d'autant plus efficaces que l'état sanitaire des animaux est plus mauvais (ro5). 
En contrepartie, on peut faire valoir les observations suivantes:

Certains antibiotiques à grand pouvoir bactériostatique n'ont aucune efficacité nutritionnelle. C'est le cas, entre autres, pour la chloromycétine, la néomycine, etc. (3I - 439-449).

Certains bactéricides très puissants, comme le succynilsulfathiazole et le phtalylsulfathiazole, diminuent la croissance du Porc et du Poulet $(49-\mathrm{I} 27-\mathrm{I} 29-25 \mathrm{I})$.

$2^{\circ}$ Dans le même état d'esprit, on a attribué aux antibiotiques efficaces une action spécifique sur la flore du tube digestif. Ils agiraient selon plusieurs modalités :

\section{A. - En favorisant la synthèse de métabolites essentiels.}

Ce serait une façon d'expliquer le rôle de facteur d'épargne que jouent les antibiotiques (273) pour certaines vitamines B. Sous leur influence, une déficience alimentaire du régime serait compensée par une plus grande élaboration bactérienne de vitamines B. L'animal serait ravitaillé par le jeu des réfections au lieu de l'être per os.

Cette hypothèse cadre mal avec 1'opinion, souvent formulée $(32-$ $70-215-28 \mathrm{r}$ ), mais également combattue (69) que les antibiotiques augmentent le besoin vitaminique du seul fait qu'ils stimulent la croissance. En outre, l'influence des antibiotiques se manifeste également avec des régimes complets et même surchargés en vitamines par addition de levure (42I) ou de foie (254).

\section{B. - En diminuant le nombre de bactéries prédatrices qui vivent en compétition avec l'hôte.}

Les études bactériologiques n'apportent aucune preuve formelle à 1 'appui de cette hypothèse $\left(4-\mathrm{I} 9-\mathrm{I} 4 \mathrm{I}-\mathrm{I}_{43}-\mathrm{I}_{44}-\mathrm{I}_{4} 6-\mathrm{I} 90-\right.$ 374). Les variations spontanées du nombre et de la nature des bactéries du tube digestif sont trop étendues d'un animal à l'autre, d'un régime à l'autre, d'une époque à l'autre $(27 \mathrm{I}-459)$, pour qu'on puisse établir un équilibre de référence de la microflore intestinale.

Faute de standard, il est donc impossible de démontrer une intervention éventuelle des antibiotiques sur la composition de la flore. Les recherches qui ont été faites dans ce sens aboutissent à une confusion complète (63). Par exemple, les coli tantôt diminuent $(5-308)$, tantôt augmentent $(3-65-353)$ sous leur influence. De plus, on sait que 1 'ingestion de certains bacilles vivants, sélectionnés comme utiles $(B$. coli, 
Bacilles lactiques $\left(5-6-35^{8}\right)$ ), ne modifient pas le taux de croissance du Poulet. Les antibiotiques favorisent le développement de $B$. coli dont le besoin en vitamine $\mathrm{BI}_{\mathrm{I}}$ entre en compétition avec le besoin de 1'hôte (I3I) ; cela ne les empêche pas de stimuler la croissance des animaux. Inversement, la sulfaméthiazine inhibe la pousse de $B$. Coli, mais n'a cependant pas d'effet sur la croissance des volailles (4I8).

Par conséquent, on ignore, d'une part, si les antibiotiques exercent une action spécifique sur la microflore; en admettant même qu'elle existe rien ne prouve, d'autre part, qu'il s'agit d'une sélection favorable.

\section{C. - En détruisant les microorganismes nuisibles qui secrètent des toxines (I 45 ).}

De fait, l'analyse des fèces montre que l'ingestion d'antibiotiques diminue le nombre des bacilles responsables de l'entérotoxémie des ovins (389), à savoir Clostridium perfringens (295).

Cette observation ne postule en rien du rôle nutritionnel des antibiotiques, car on n'a pas pu mettre en évidence 1'existence de la moindre relation entre le nombre des Clostridium dans les fèces et le taux de croissance des porcs $(438)$ et des volailles $(228-259)$. De plus, on ne retrouve pas en général ces formes microbiennes dans les fèces du Porc et l'administration d'antitoxine de $C l$. perfringens n'a pas d'effet de croissance sur le Poulet.

On a également invoqué le fait que de fortes doses d'auréomycine préviennent la nécrose hépatique provoquée par une forte ingestion de levure (I9I - I92). Cette prévention est attribuée à l'inhibition de la secrétion de toxine microbienne ou à la destruction des microbes euxmêmes (92) ; mais la preuve de cette assertion manque.

Pour résumer ce paragraphe et en souligner la complexité, rapportons en conclusion deux notions fondamentalement opposées quant aux relations éventuelles entre antibiotiques et flore intestinale.

D'après plusieurs auteurs, l'administration parentérale d'antibiotiques ou leur implantation sous forme de "pellets " n'aurait aucun effet sur la croissance du Porc et du Rat (34 - 6I - I6I - I89 - 354).

D'après d'autres auteurs, tout aussi affirmatifs, l'injection d'antibiotiques serait aussi efficace que 1'ingestion (3I - 343 - 427).

Il paraît donc prématuré de tirer des conclusions à partir de données si disparates. D'autant plus que la voie d'administration ne conditionne nullement la présence des antibiotiques dans le tube digestif où ils peuvent pénétrer par la bile et même par simple absorption à partir du sang $\left(25^{8}\right)$. 
$3^{\circ}$ Beaucoup plus séduisante est la théorie anglaise dite théorie " d'Infection " qui repose sur la notion de microbisme latent. Elle repose sur des faits complexes et sur plusieurs observations expérimentales, dues notamment à COATEs et col. (IOI - IO3 - I05 - I26) :

- les éleveurs de volailles nouvellement installés obtiennent de meilleurs résultats que leurs confrères anciennement établis;

- 1'action de croissance des antibiotiques est d'autant plus marquée que les conditions sanitaires sont plus mauvaises;

- ces corps n'ont pas d'efficacité, si on les administre à des poussins élevés stérilement (350).

On était ainsi conduit à penser qu'au bout d'un certain temps, les locaux d'élevage étaient nécessairement "infectés " par un microbisme latent (FERRANDO (I55)), dont la manifestation invisible sur le plan pathologique, se traduisait par une croissance retardée. En réduisant l'incidence de ce que Catron $(88-89)$ appelle " le niveau de maladie", les antibiotiques agiraient dès lors nécessairement sur le taux du développement.

Cette thèse a connu une grande faveur depuis les expériences conjuguées de READING-GLAXo. Le même antibiotique administré à des poulets de même race, soumis à des régimes identiques, s'est montré inefficace au Laboratoire de GLAxo et efficace au Laboratoire de REAding. La seule variable dans ces essais concernait le poulailler, local expérimental nouvellement construit pour les essais de GLAxo, ancien poulailler d'usage et d'élevage à l'Institut de READING (I03 - I05).

Contre-épreuve : READING construit un local neuf et dès lors les antibiotiques n'ont plus d'efficacité.

I a notion de microbisme latent est à l'ordre du jour tant pour les poulaillers que pour les porcheries (FERRANDO) (I55). Justifie-t-elle pleinement l'action nutritive des antibiotiques?

Tout d'abord, il faut reconnaître qu'elle repose sur peu d'observations $(220-270)$. Si des élevages récents donnent parfois de meilleurs résultats que les élevages anciens, on ne saurait toutefois prétendre que l'ancienneté des locaux conditionne automatiquement la vitesse de croissance des animaux. De plus, cette théorie que l'on peut soutenir en ce qui concerne la croissance rend difficilement compte de l'amélioration de l'indice de consommation sous l'effet des antibiotiques. Enfin et surtout, elle est battue en brèche par les expériences de HILL (2I9), de BARTLEY sur veaux (25) et par la nouvelle technique avicole, dite "des litières accumulées " (I93 - I94-242-243).

Les éleveurs obtiennent, en effet, des résultats excellents en maintenant au poulailler une litière continue à laquelle on ajoute périodiquement une couche superficielle propre.

Il semble que ces conditions assurent au Poussin la fourniture de 
facteurs de croissance élaborés par la flore des litières accumulées. Ces litières, par contre, créent et entretiennent le milieu le plus favorable au microbisme latent puisqu'elles récoltent les déjections de tous les sujets, sains et malades, et interdisent les mesures d'hygiène habituelles. Il faut, en outre, signaler que le plein effet des litières accumulées ne se fait sentir qu'après un certain temps. Or, ce temps de latence correspond justement à la durée nécessaire à l'installation du microbisme des litières.

$4^{0}$ L'effet nutritionnel des antibiotiques s'expliquerait par une modification de la perméabilité cellulaire et une meilleure absorption intestinale. Cette théorie est fondée sur des analogies et des observations directes.

Certains composés arsenicaux augmentent la croissance du Dindon (309). Il en est de même de 1'acide 3-nitro-4-hydroxyphényl-arsenique qui améliore la croissance du Poulet (47) et du Porc $(259-28 \mathrm{I}-377)$. BIRD, sur Poulet (45), obtient même des résultats supérieurs à ceux des antibiotiques. Or, ces corps ont des propriétés tensio-actives et agissent par là-même sur la perméabilité cellulaire.

Quelques antibiotiques étant également tensio-actifs, on leur a appliqué les raisonnements précédents. A l'appui de cette hypothèse, on a fait valoir que l'auréomycine, traitée à la soude N/Io, perd son pouvoir bactériostatique, mais demeure tensio-active et garde son efficacité pour la croissance du Poussin (455), du Dindon (4I6) et du Porc (83).

C'est également à des modifications de la perméabilité cellulaire ( $109-\mathrm{I}_{52}$ ), qu'on a voulu rapporter certains effets des antibiotiques :

- meilleure utilisation du radiocalcium pour l'ossification du Poulet sous l'effet de la pénicilline $(74-$ II $3-298)$;

- traitement du perosis dû à la déficience en manganèse, par la pénicilline (397) et l'auréomycine $(334-456)$;

- efficacité plus grande du carotène et épargne des vitamines A et $\mathrm{D}$ en présence d'antibiotiques ( $18-73-\mathrm{I} 57-207-209-347-$ $408)$;

- renforcement de l'hyperglycémie après ingestion de glucose sous l'effet des antibiotiques.

L'interprétation de ces faits, dont certains ont été contestés (I08 207), met en cause une amélioration de l'absorption intestinale par les antibiotiques.

Dans le même sens, on peut également rapporter les faits suivants :

- l'ingestion d'antibiotiques se traduit sur la morphologie de l'intestin grêle: sa longueur augmente (I8I), son poids diminue (463), l'épaisseur de la barrière intestinale s'amenuise ( 139 - I8r), toutes causes favorables à la résorption des nutriments ; 
- in vitro, et travaillant sur anse intestinale isolée, FERRANDo (I56) démontre que l'auréomycine augmente la perméabilité de la paroi intestinale à l'azote.

Ce dernier résultat a été obtenu avec des doses d'antibiotiques très supérieures aux quantités habituellement utilisées.

A cet ensemble de faits qui permettrait d'expliquer l'action nutritionnelle des antibiotiques sous l'angle d'une meilleure utilisation digestive, on peut opposer un ensemble de faits qui vont à l'encontre de cette conception.

L'efficacité des antibiotiques inactivés par la soude a été mise en doute par de récents travaux ( $5_{5} 8-\mathrm{I}_{59}-\mathrm{I}_{72}$ ).

In vivo, de très nombreux auteurs ont insisté sur le fait que les antibiotiques ne modifiaient pas la digestibilité de la ration totale, ni le coefficient d'utilisation digestive (C. U. D.) azoté (97). (Nous arriverons à la même conclusion dans nos propres recherches).

Enfin, l'assimilation des effets des antibiotiques à ceux des corps tensio-actifs est critiquable sur plus d'un point.

Les tensio-actifs n'entraînent pas nécessairement une amélioration de la croissance $\left(33-\mathrm{I}_{53}-384-385-4 \mathrm{r} 4\right)$.

Sur I5 corps tensio-actifs éprouvés, ẾLY (I48) n'en rencontre que quatre qui agissent favorablement sur le développement. De plus, ils n'ont pas d'action sur l'utilisation des vitamines B (I74). Enfin, leur action n'est pas reproductible d'un essai à l'autre $(204-377-4 \mathrm{I} 4)$. Des études faites au Laboratoire de Bellevue concluent dans le même sens : l'incorporation au régime du Rat d'un émulsifiant à fortes propriétés tensio-actives ne se manifeste ni sur le plan de la croissance, ni sur celui de l'utilisation digestive.

Enfin, les modalités d'action des antibiotiques et des corps tensioactifs, reconnus efficaces, sont différentes. Les premiers ont un effet d'autant plus grand que l'animal est plus jeune ; l'influence des seconds est indépendante de 1'âge des animaux $(35-46-$ I 49 - I86).

$5^{0}$ Une dernière théorie très vague fait intervenir les antibiotiques au niveau du métabolisme endocrinien. Elle fait état des observations suivantes :

- l'injection de cortisone provoque des troubles divers (diminution de la croissance, chute de cheveux, involution du thymus) que prévient l'administration d'auréomycine avec ou sans vitamine BI2 (293);

- BARNARD et ERSHOFF ( 2 I - 22-I5I) reconnaissent à l'auréomycine une action adrénocorticomimétique. On a objecté à leur théorie que 1'hyperplasie des surrénales n'est pas directement attribuable aux 
effets de 1'antibiotique, mais la conséquence secondaire de l'augmentation du poids corporel (I34);

- dans le diabète à l'alloxane, l'auréomycine améliore la croissance des animaux (IO9). Cet effet est consécutif à une augmentation de 1'appétence. Peut-on dès lors parler de métabolisme endocrinien?

Les arguments en faveur d'une théorie hormonale du rôle des antibiotiques sont donc assez faibles en eux-mêmes. On peut, en outre, leur opposer d'autres résultats expérimentaux qui vont à l'encontre de 1'hypothèse.

L'auréomycine n'a aucun effet sur la morphologie de la thyroïde (264) et n'intervient pas dans l'action de la thyroxine (20).

Même observation pour les gonades dont la structure n'est pas modifiée par la terramycine (IO9 - I34). Les antibiotiques demeurent sans action sur la gestation (84), la lactation $(27-84-201-319)$ et la ponte $(86-268-290-446)$. Enfin, ils n'affectent pas les performances des reproducteurs mâles (79).

Il faut cependant signaler une particularité liée au mode d'administration.

L.e transfert au lait et à l'œuf des antibiotiques utilisés per os aux doses habituelles est assez faible. Les jeunes à la mamelle n'en retirent pas de bénéfice alors qu'ils réagissent favorablement à l'enrichissement direct $\mathrm{du}$ lait en antibiotiques (I38). Par contre, l'emploi thérapeutique d'antibiotiques dans le traitement des mammites (crayons à la pénicilline notamment) se répercute au niveau du lait (252) qui devient impropre à la fabrication des fromages en raison de sa teneur en principes bactériostatiques (I4 - 329-453-457).

I1 n'existe donc aucun argument probant en faveur des relations entre antibiotiques et hormones.

Que ressort-il de cette brève étude des effets nutritionnels et des modalités d'action des antibiotiques?

On ne peut en tirer aucune conclusion d'ensemble ni quant à la reproductibilité des faits expérimentaux, ni quant à la validité de telle ou telle hypothèse relative aux mécanismes mis en jeu.

\section{III. - ORIENTATION DES RECHERCHES ENTREPRISES}

Devant 1'étendue et la complexité des problèmes soulevés par l'usage alimentaire des antibiotiques, il est donc impossible de se faire actuellement une idée de leur rôle physiologique et des mécanismes par lesquels ils agissent. Notre intention, en entreprenant des recherches dans cette voie, n'était pas de résoudre les nombreux points demeurés 
obscurs. Beaucoup plus modestement, nous nous sommes proposés, d'une part, de procéder à certaines vérifications, d'autre part, de poursuivre des investigations nouvelles. Cela nous a conduit à progresser étape par étape.

Dans le premier chapitre de notre étude, nous montrerons le pouvoir d'épargne des antibiotiques vis-à-vis des vitamines $B$. Ce rôle avait déjà été établi par des études sur la croissance. Nous en préciserons la signification par le dosage des réserves vitaminiques du foie.

Dans le second chapitre, des dosages directs sur le contenu vitaminique des fèces nous permettront d'écarter l'hypothèse selon laquelle les antibiotiques agissent au niveau de la flore intestinale en favorisant la synthèse des vitamines $B$.

Dans le chapitre III, nous établirons la signification des relations entre vitamine $\mathrm{BI} 2$ et antibiotique et montrerons qu'il n'y a aucune possibilité d'interchangeabilité entre ces deux substances. Nous serons également conduits à étudier le rôle nutritionnel de la vitamine Brz.

Dans le chapitre IV, l'établissement des bilans nous permettra de savoir si les antibiotiques influencent les facteurs qui conditionnent 1'efficacité alimentaire, à savoir le coefficient d'utilisation digestive et le coefflcient de rétention métabolique.

Cette étude sera précisée dans le chapitre V par l'analyse corporelle des animaux. Nous serons ainsi amenés à montrer chez le Rat que l'auréomycine agit spécifiquement sur un seul élément constitutif de la carcasse et que cette action explique, à elle seule, la stimulation de croissance pondérale.

Notre travail n'a donc pas la prétention d'expliciter le mode d'action des antibiotiques en général, mais uniquement de préciser le rôle nutritionnel d'un antibiotique donné et la signification du bénéfice pondéral qu'il provoque. 
CHAPITRE PREMIER

\section{EFFET D'EPARGNE DES ANTIBIOTIQUES POUR LES VITAMINES DU GROUPE B}

\section{I. - INFLUENCE DES ANTIBIOTIQUES SUR LA RÉSERVE VITAMINIQUE DU FOIE}

\section{PAR}

\section{CALET ET A. RERAT}

\section{A. - Position du problème.}

Les besoins vitaminiques du jeune sont très élevés et d'autant plus impérieux que la croissance est plus forte. A priori, les antibiotiques qui augmentent le développement pondéral devraient donc accroître les exigences vitaminiques. C'est ce que soutiennent les auteurs qui estiment que 1e plein effet des antibiotiques exige un enrichissement de la ration en vitamines $B(32-69-70-215-28 \mathrm{I})$.

En fait, cette notion n'a pas été confirmée par les nombreuses études poursuivies dans le but de préciser les relations entre antibiotiques et vitamines B. Ici aussi les résultats sont contradictoires.

D'un côté, certains auteurs ( $\mathrm{IO} 2-\mathrm{I} 28-4 \mathrm{I} 9-445$ ), utilisant des régimes synthétiques carencés spécifiquement en telle ou telle vitamine, constatent que les antibiotiques sont inopérants à remédier aux déficiences vitaminiques. C'est en particulier le cas pour l'acide pantothénique $(189-279-370)$, la pyridoxine $(34 \mathrm{I}-342)$, la nicotinamide $(3 \mathrm{I} 7-400)$, etc.

A l'opposé, on a soutenu, à la suite d'observations portant sur la croissance, que les antibiotiques exercent une action d'épargne vis-à-vis des vitamines $B$, en ce sens qu'ils favorisent le développement des animaux recevant un apport vitaminique à taux suboptimal (I64-307$334-37 x-398)$.

Ainsi un régime subcarencé en vitamine $\mathrm{B} 2$ est valorisé aussi bien par des antibiotiques que par enrichissement en riboflavine (267).

La pénicilline et à un degré moindre l'auréomycine remédient partiellement à la déficience alimentaire en vitamine BI au même titre que l'injection de thiamine (443).

Normalement l'efficacité de la pyridoxine est inférieure à celle du pyridoxal; la présence d'auréomycine annule cette différence (273).

Les faits sont moins évidents pour d'autres vitamines. Pour certains auteurs, les antibiotiques ont un effet d'épargne pour l'acide fo- 
lique $(43-50-$ I02 - 307 - 447). Pour d'autres, il n'en serait rien et, bien plus, l'auréomycine déprécierait la croissance du Poulet $(67-$ 68 - 277) et du Rat (I29 - 32I - 462), carencés en acide folique. De très nombreuses études ont été poursuivies en relation avec la vitamine Br2. Les résultats, ici aussi, peuvent se grouper en positifs et négatifs. On a soutenu que les antibiotiques amélioraient la croissance (II7 - 203), diminuaient la mortalité (339) et réduisaient 1'incidence des anémies pernicieuses chez les poulets carencés en $\operatorname{Br} 2(265-440)$. Pourtant, on a également observé le contraire avec le Porc (90) et chez le Dindon (386). Enfin, l'effet synergique entre antibiotique et BI2 signalé par la majorité des auteurs va à l'encontre de la notion d'épargne (3I9 $326-4 \mathrm{I} 8$ ).

Nous sommes donc en présence de deux grandes thèses exclusives l'une de 1'autre: soit que les antibiotiques augmentent le besoin en vitamines $B$, soit qu'ils le diminuent, en vertu d'une action d'épargne.

Devant de telles contradictions, nous avons tenu à entreprendre des recherches personnelles dans le but de savoir dans quelle mesure les antibiotiques pouvaient parer à une déficience alimentaire en vitamines $B$. Pour être significative, cette investigation nécessitait et le choix du rat comme réactif animal et, par voie de conséquence, non seulement des modalités expérimentales rigoureuses, mais aussi l'adoption de critères valables. La réponse de croissance, choisie par tous les auteurs précédents, nous a semblé insuffisante pour établir la notion d'économie des vitamines $B$ par les antibiotiques.

I1 nous a paru nécessaire d'étayer le test basé sur le gain de poids par la mesure des réserves vitaminiques de l'organisme. Ainsi, nous avons été conduits à doser les réserves hépatiques de riboflavine, acide pantothénique, nicotinamide et vitamine BI2.

\section{B. - Conditions expérimentales.}

Le régime de base identique pour tous les lots était le suivant :

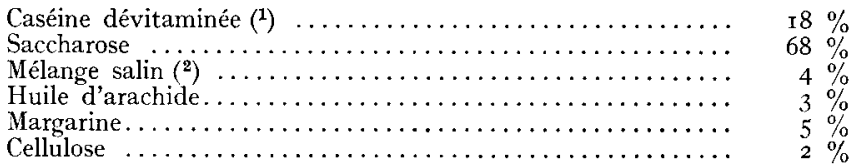

(1) La caséine est dévitaminée par des traitements successifs à l'alcool, la soude à I,2 p. Ioo et l'acide acétique à 2 p. 100.

(2) Le mélange salin est celui d'Osborne et Mendel modifié par Lafon (M.) (I939). Thèse Doct. ès-Sc. PARIS : il contient en grammes :

Carbonate de potassium ......... 565,2

Carbonate de calcium ............... $53.5 \%$

Carbonate de sodium............ I 36,8

Carbonate de magnésium ........ 96.6

Sesquicitrate ferrique............. $\quad 25,36$

Acide orthophosphorique.......... $44_{412,0}$

Acide chlorhydrique.......... 2 2 3,6

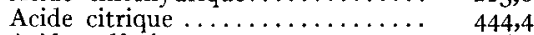

Acide sulfurique $\ldots \ldots \ldots \ldots \ldots \ldots . \quad 36,8$

Iodure de potassium ............

Sulfate de manganèse .......... 0,632

Fluorure de sodium........... 0,96

Alun de potassium cristal........ 
Il contenait en plus les éléments suivants pour I $\mathrm{kg}$ :

Vitamine $\mathrm{E}$

Cystine $1(-)$

$30 \mathrm{mg}$

Vitamine $\mathrm{K}$

$5 \mathrm{mg}$

Les vitamines $A$ et $D$ étaient apportées par une huile vitaminée (A : I20 o0o U. I./cc et D : Io 000 U. I./cc), à raison de 4 gouttes par semaine et par rat.

Les lots expérimentaux sont ainsi composés :

\begin{tabular}{|c|c|c|c|c|c|}
\hline No des lots & $\begin{array}{c}\text { Nombre } \\
\text { d'animaux }\end{array}$ & $\underset{\text { base }}{\text { Régime de }}$ & $\begin{array}{l}\text { Mélange de } \\
\text { vitamines B }\end{array}$ & Auréomycine & Pénicilline \\
\hline $\begin{array}{l}\text { Témoins } \mathrm{N} \ldots \ldots \ldots \ldots \ldots \\
\text { Carencés } \mathrm{C} \ldots \ldots \ldots \ldots \ldots \\
\text { Carencés }+ \text { Auréomycine Au. } \\
\text { Carencés }+ \text { Pénicilline P } . .\end{array}$ & $\begin{array}{l}6 \\
9 \\
9 \\
9\end{array}$ & $\begin{array}{l}+ \\
+ \\
+ \\
+\end{array}$ & $\begin{array}{ll}\text { I } & \% \\
0, \mathbf{I} & \% \\
0, \mathbf{I} & \% \\
\text { O, I } & \%\end{array}$ & Ioo $\overline{\mathrm{mg}} / \mathrm{kg}$ & $\frac{\overline{-}}{100 \mathrm{mg} / \mathrm{kg}(3)}$ \\
\hline
\end{tabular}

(3) Soit r60 $\mathrm{mg} / \mathrm{kg}$ de pénicilline-procaïne.

Le mélange de vitamines $\mathrm{B}$ a la composition suivante, en $\mathrm{g}$ :

Inositol

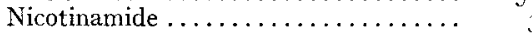

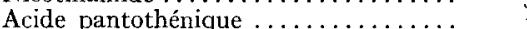

Thiamine ....................

Riboflavine $\ldots \ldots \ldots \ldots \ldots \ldots \ldots \ldots$

Pyridoxine

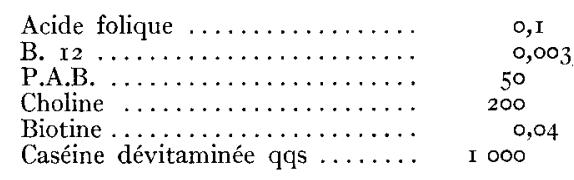

A la dose de I p. Ioo, ce mélange correspond aux normes optimales des vitamines $B$ pour le Rat en croissance. A la dose de o, I p. Ioo, il réalise un état de déficience chronique, mais non de carence aiguë. Nous nous sommes assurés, par dosage direct, que dans les conditions où nous l'ajoutions au régime, l'antibiotique demeurait stable et non détruit. Il a été reconnu en effet (72) que les antibiotiques peuvent se dégrader vite.

Les animaux mangeaient ad libitum. Ils étaient pesés régulièrement ainsi que les quantités de nourritures ingérées. L'expérience a duré 66 jours.

\section{C. - Résultats expérimentaux.}

\section{a) Observations sur animal.}

Du point de vue état général rien à signaler dans le lot $\mathrm{N}$ (témoins). Signes carentiels certains dans le lot C : alopécie, diarrhée, porphynurie pileuse. La pénicilline (lot $\mathrm{P}$ ) remédie aux diarrhées et prévient 1'alopécie. Au contraire, l'auréomycine (lot Au) aggrave l'alopécie et le dépôt de porphyrine.

Les résultats portant sur la croissance sont condensés dans le tableau I. 
Tableau I

\begin{tabular}{|c|c|c|c|c|}
\hline Lots & $\mathbf{N}$ témoins & C carencés & $\mathbf{A} \mathbf{u}=\mathbf{C}+$ Auréo & $\mathbf{P}=\mathrm{C}+$ Pénicil \\
\hline Poids à la mort $(g) \ldots \ldots$. & $2 \mathrm{I} 7,0$ & I 17,5 & I 53,5 & I 35,5 \\
\hline Gain de poids total $(g) \ldots$. & 169,0 & 70,0 & 107,0 & 90,0 \\
\hline Ecart-type du gain de poids.... . & 22,2 & 28,3 & 32,6 & 31,7 \\
\hline Gain de poids journalier $(\mathrm{g}) \ldots \ldots$ & 2,5 & $\mathrm{I}, \mathrm{I}$ & 1,6 & $\mathrm{I}, 4$ \\
\hline Nourriture ingérée par jour $(\mathrm{g})$. . & 667,0 & 454,0 & 566,0 & 500,0 \\
\hline Indice de consommation. . . . . . . & 3,9 & 6,5 & 5,3 & 5,7 \\
\hline Poids des foies $(\mathrm{g}) \ldots \ldots \ldots \ldots$ & 7,4 & 4,9 & 7,3 & 6,2 \\
\hline$\frac{\text { Poids des foies }}{\text { Poids vif }} \times 100 \ldots \ldots$. & 3,4 & 4,9 & 4,7 & 4,2 \\
\hline $\begin{array}{l}\text { Poids vif } \\
\text { Ecart-type de ce rapport. .... }\end{array}$ & $0,5^{2}$ & $0,5 \mathrm{I}$ & $\mathrm{I}, \infty \mathrm{O}$ & 0,77 \\
\hline
\end{tabular}

On voit que :

- le ralentissement de croissance provoqué par la subcarence en vitamines B peut être partiellement compensé par un antibiotique donné. Dans nos essais, la différence de gain de poids entre les lots $C$ (carencé) et $\mathrm{P}$ (à la pénicilline) n'est pas significative (probabilité $>20 \mathrm{p}$. Ioo) alors qu'elle l'est hautement entre les lots C (carencés) et Au (à l'auréomycine) : l'augmentation du gain de poids due à l'auréomycine est de 49 p. roo;

- l'écart-type du lot témoin (N) est faible; il est beaucoup plus important dans tous les lots carencés. Cette observation va à l'encontre de la notion pratique qui veut que l'auréomycine "égalise les lots";

- le bénéfice apporté par l'auréomycine ne remédie pas pleinement à la carence en vitamines $B$, puisque la différence des gains de poids est hautement significative entre les lots $N$ (témoins normaux) et $\mathrm{Au}$ (carencés à l'auréomycine) ;

- les antibiotiques employés ne modifient pas la cinétique de la croissance : la carence en vitamines $\mathrm{B}$ s'accompagne toujours à la quatrième semaine d'une phase critique dans le taux de la croissance relative (pourcentage quotidien de gain de poids). Cette phase critique se retrouve avec l'auréomycine qui ne fait qu'en atténuer la gravité (fig. 2);

- l'auréomycine augmente de 27 p. roo le taux d'ingestion des animaux carencés, alors que le taux de croissance est amélioré de 43 p. Ioo. Il en résulte que cet antibiotique agit directement sur la valeur de l'indice de consommation (quantité de nourriture sèche ingérée pour un gain de poids vif de I g) en le diminuant de façon significative. Toutefois, l'indice de consommation est encore plus bas pour les lots normaux en régime équilibré ;

- les antibiotiques semblent nettement augmenter la masse relative du foie par unité de poids vif. En observation macroscopique directe le fait ne paraît pas imputable à une stéatose : foies roses sans pigmentation grasse. 


\section{b) Réserves vitaminiques.}

Les résultats des dosages de nicotinamide, riboflavine, acide pantothénique et vitamine BI2 se trouvent dans le tableau suivant:

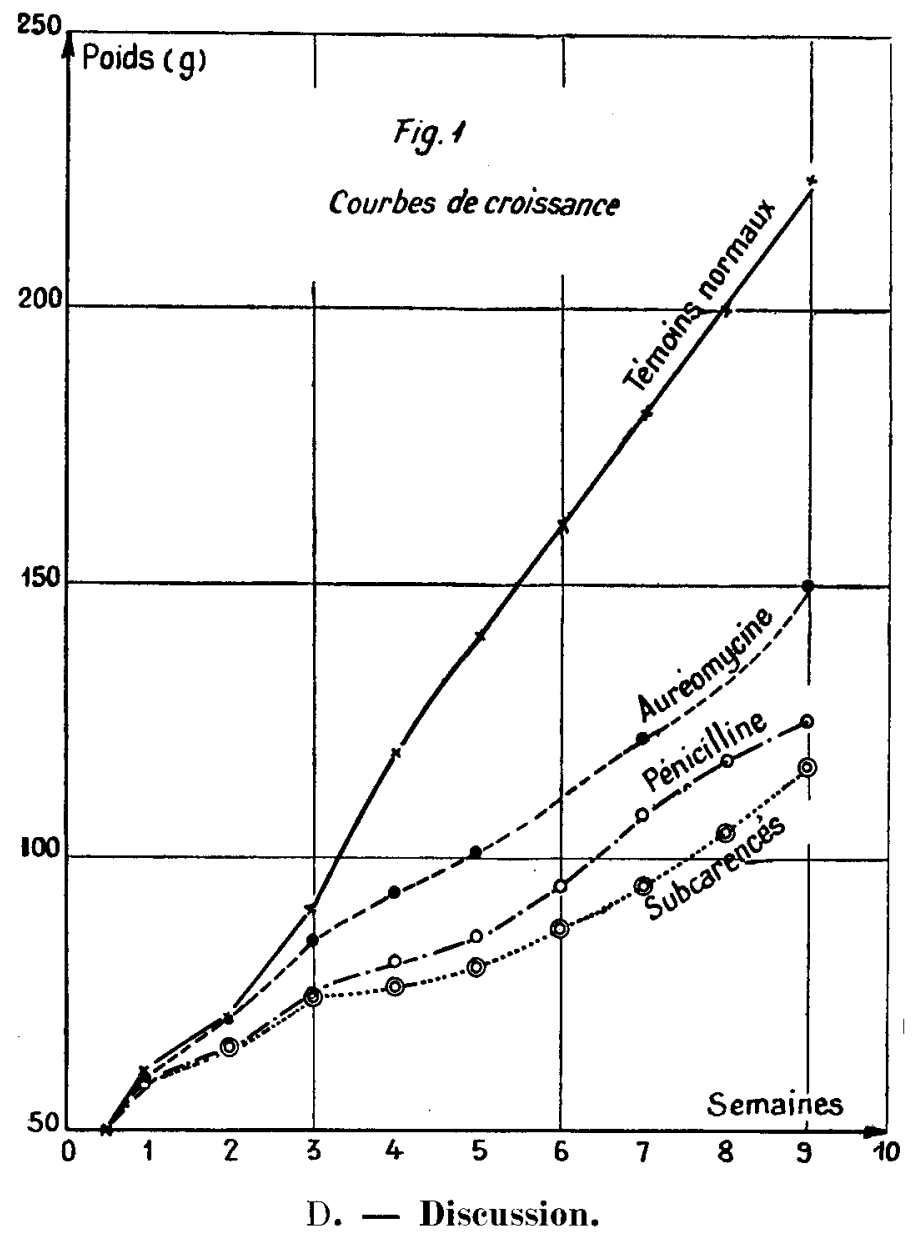

Si les antibiotiques permettent une meilleure croissance en régime carencé, ils ne remédient cependant pas complètement aux effets des déficiences vitaminiques.

Les rôles des différentes vitamines B sont complexes et il est difficile de relier étroitement la vitesse de croissance et le taux des réserves hépatiques. Toutefois, une discrimination peut être faite.

Pour la vitamine $\mathrm{BI} 2$, incontestablement les antibiotiques favorisent sa mise en réserve, quel qu'en soit le mode d'expression : pourcentage, teneur hépatique totale en valeurs absolues ou relatives. Le pouvoir d'épargne senble certain puisque les animaux carencés soumis aux antibiotiques ont des foies plus riches que les témoins. Cet effet spécifique 


\section{Tableau II}

Constitution vitaminique des foies.

\begin{tabular}{|c|c|c|c|c|}
\hline $\begin{array}{l}\text { Vitamine } \\
\text { dosée }\end{array}$ & Régime & $\begin{array}{l}\text { Teneur en } \gamma \\
\text { par } g \text { foie frais }\end{array}$ & $\begin{array}{l}\text { Teneur en Y' } \\
\text { par foie entier }\end{array}$ & $\begin{array}{c}\text { Teneur vitaminique } \\
\text { du foie ramenée } \\
\text { à Ioog de poids } \\
\text { vif en } \gamma\end{array}$ \\
\hline PP Nicotinamide & $\begin{array}{l}\text { Normal } \\
\text { Subcarencé } \\
\text { + Pénicilline } \\
\text { + Auréomycine }\end{array}$ & $\begin{array}{l}\text { I } 66 \\
\text { I } 60 \\
\text { I } 4 I \\
\text { I } 44\end{array}$ & $\begin{array}{r}\text { I } 217 \\
77 \mathrm{I} \\
875 \\
\text { I } 029\end{array}$ & $\begin{array}{l}56 \mathrm{I} \\
670 \\
660 \\
694\end{array}$ \\
\hline Riboflavine & $\begin{array}{l}\text { Normal } \\
\text { Subcarencé } \\
+ \text { Pénicilline } \\
+ \text { Auréomycine }\end{array}$ & $\begin{array}{l}40,5 \\
33,3 \\
22,5 \\
27,0\end{array}$ & $\begin{array}{l}302,5 \\
162,5 \\
138,0 \\
192,0\end{array}$ & $\begin{array}{l}137,5 \\
139,0 \\
104,0 \\
128,0\end{array}$ \\
\hline $\begin{array}{l}\text { Acide } \\
\text { pantothénique }\end{array}$ & $\begin{array}{l}\text { Normal } \\
\text { Subcarencé } \\
+ \text { Pénicilline } \\
\text { + Auréomycine }\end{array}$ & $\begin{array}{l}75 \\
85 \\
73 \\
65\end{array}$ & $\begin{array}{l}546 \\
395 \\
45^{2} \\
474\end{array}$ & $\begin{array}{l}250 \\
342 \\
338 \\
320\end{array}$ \\
\hline Vitamine $B_{12}$ & $\begin{array}{l}\text { Normal } \\
\text { Subcarencé } \\
\text { + Pénicilline } \\
\text { + Auréomycine }\end{array}$ & $\begin{array}{l}0,12 \\
0,12 \\
0,20 \\
0,14\end{array}$ & $\begin{array}{l}0,86 \\
0,60 \\
+, 30 \\
1,03\end{array}$ & $\begin{array}{l}0,39 \\
0,52 \\
0,82 \\
0,70\end{array}$ \\
\hline
\end{tabular}

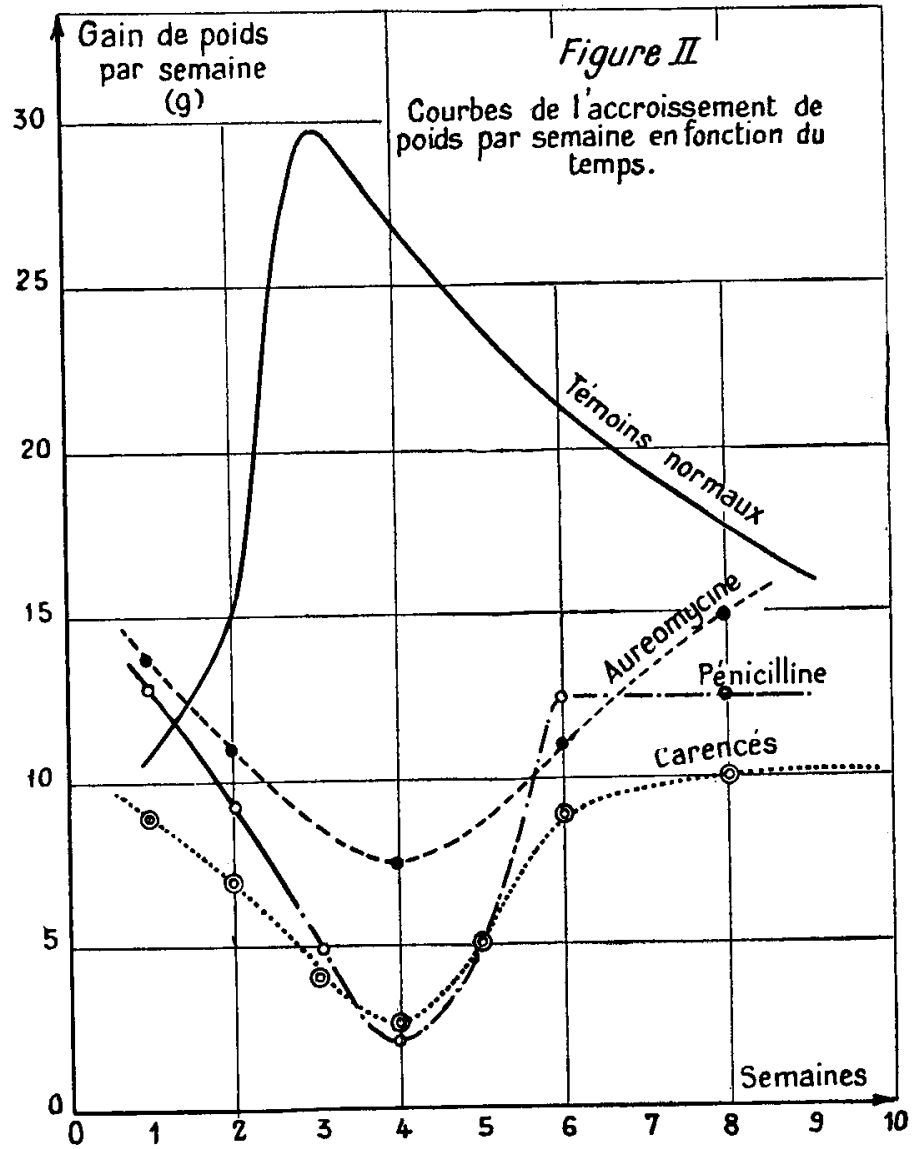


sur le métabolisme de la vitamine Br2 pourrait peut-être expliquer à lui seul l'influence favorable des antibiotiques sur la croissance. Nous en discuterons par la suite.

En ce qui regarde la nicotinamide, la riboflavine et l'acide pantothénique, les commentaires sont plus nuancés. Les chiffres du tableau II montrent que même l'étude du foie ne permet pas de préciser le rôle complexe des antibiotiques vis-à-vis des vitamines B. On peut simplement noter que l'auréomycine augmente le potentiel vitaminique total $\mathrm{du}$ foie en accroissant la masse hépatique. L'augmentation du poids du foie déjà notée (463) et l'augmentation de la teneur du foie vérifiée par plusieurs auteurs (I $89-267-307)$ ne permettent pas de conclure à la notion de pouvoir d'épargne.

En fait, ni l'augmentation du taux de croissance, ni la rétention accrue de vitamines dans les tissus ne sont des tests suffisants à démontrer qu'en régime subcarencé les antibiotiques épargnent les vitamines B. Il faudrait de plus qu'ils rétablissent certaines fonctions physiologiques altérées par la carence. Si certaines observations vont à l'appui de cette thèse, d'autres laissent subsister des doutes quant à cette possibilité : tout d'abord la bacitracine améliore le poids et le plumage des volailles carencées en acide folique (306). Les antibiotiques favorisent les performances des reproducteurs (80). Enfin des doses élevées d'auréomycine relèvent le taux de ponte des élevages peu productifs et la pénicilline ou la terramycine, à dose normale, rehaussent de 6 à i 8 p. Ioo le taux de l'éclosabilité des oufs de poules déficientes en vitamine BI2, comme le fait 1'administration de BI2 elle-même.

Par contre, d'autres auteurs nient ces résultats et pensent que les antibiotiques ne remédient pas à certains troubles consécutifs à la carence en vitamine BI2 : chute des érythrocites et de l'hémoglobine, diminution du taux d'éclosion de l'œuf.

Le dilemme est donc le suivant: en régime subcarencé, les antibiotiques semblent exercer une action d'épargne vis-à-vis des vitamines $B$ si on choisit la croissance et les réserves vitaminiques comme test, mais cette action ne s'inscrit pas nécessairement dans le cadre des fonctions physiologiques altérées par la carence.

Pour affirmer l'existence ou l'absence d'un pouvoir d'épargne, il faudrait donc disposer d'une unité de référence valable. Nous avons choisi pour cela des éléments cellulaires caractéristiques, à savoir les acides nucléiques et l'azote protéique.

L'étude du rapport $\frac{\text { vitamine }}{\text { acides nucléiques }}$ est donc nécessaire pour mettre en évidence le pouvoir d'épargne des antibiotiques vis-à-vis des vitamines $B$. 


\section{II. - MISE EN ÉVIDENCE DU POUVOIR D'ÉPARGNE}

PAR

\section{CALET}

\section{$\mathrm{I}^{\circ}$ Conditions expérimentales et observations in vivo.}

Deux lots de Io rats pesant au départ $25 \mathrm{~g}$ sont soumis pendant $5^{6}$ jours aux régimes $C$ et $A u$ précédemment décrits : régimes subcarencés en vitamines $B$ au dixième des valeurs optimales sans et avec addition d'auréomycine.

L'extrême jeunesse des animaux les a rendus particulièrement sensibles aux effets de la carence B, comme le montre le tableau III.

TABLEAU III

\begin{tabular}{|c|c|c|}
\hline & $\mathrm{Au}$ & C \\
\hline 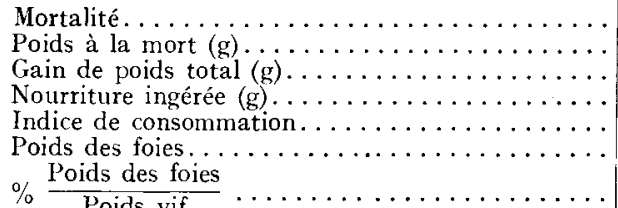 & $\begin{array}{r}20 \% \\
97 \\
65 \\
364 \\
5,6 \\
4,4 \\
4,6\end{array}$ & $\begin{array}{l}60 \% \\
90 \\
59 \\
342 \\
5,75 \\
3,9 \\
4,4\end{array}$ \\
\hline
\end{tabular}

L'auréomycine a, dans ces conditions, un effet nettement favorable sur la survie, alors que son effet est pratiquement nul sur la vitesse de la croissance. Il s'est fait dans le lot C une sorte de sélection naturelle qui n'a laissé survivre que les animaux les plus résistants et par là même les plus aptes à se développer. Cette expérience a, ainsi, l'avantage de permettre une comparaison rigoureuse entre animaux de même niveau de croissance et de poids identique au moment du sacrifice.

\section{$2^{\circ}$ Etude biochimique du foie.}

Le tableau IV est calculé d'après les teneurs du foie en vitamine BI2, azote protéique résiduel, acides désoxy et ribonucléiques.

Chez les animaux subcarencés et sous l'effet des antibiotiques, 1'azote protéique et l'acide ribonucléique du foie ne varient pas ou augmentent légèrement. Ainsi, la mesure directe de Brz du foie total est aussi démonstrative que les rapports $\mathrm{BI} / \mathrm{N}$ protéique et $\mathrm{BI} / \mathrm{R} . \mathrm{N}$.A.

$A u$ contraire, l'acide désoxy-ribonucléique diminue significativement. On peut donc dire que l'augmentation de vitamine BI2 produite par les antibiotiques est un phénomène spécifique et autonome, complète- 


\begin{tabular}{|c|c|c|}
\hline & $\mathrm{Au}$ & $\mathrm{C}$ \\
\hline $\mathrm{B}$ I $2 / \mathrm{g}$ de foie $(\mathrm{m} \%)$ & I63 & 102 \\
\hline 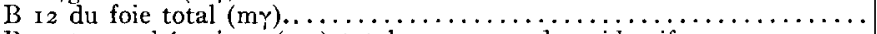 & 720 & 390 \\
\hline 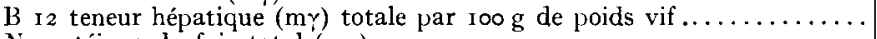 & 755 & 452 \\
\hline $\mathrm{N}$ protéique du foie total (mg)... & 126 & 126 \\
\hline R.N.A. en phosphore du foie total $(y) \ldots \ldots$. & I 867 & I $6 \mathrm{I} 8$ \\
\hline $\begin{array}{l}\text { D.N.A. en phosphore du foie total }(\gamma) \ldots \ldots \ldots \ldots \ldots \ldots \ldots \ldots \ldots \cdots \\
\text { B } 12 \times 10^{6} \text { du foie total }\end{array}$ & 671 & 796 \\
\hline $\begin{array}{l}\mathrm{N} \text { protéique du foie total } \\
\mathrm{B} \mathbf{I}_{2} \times \mathrm{IO}^{4} \mathrm{du} \text { foie total }\end{array}$ & 5,76 & 3,10 \\
\hline R.N.A. du foie total $\cdots$ & $4, \mathrm{iI}$ & $2,5^{8}$ \\
\hline B $12 \times$ I 000 du foie total & & \\
\hline D.N.A. du foie total & 1,12 & 0,505 \\
\hline
\end{tabular}

ment indépendant du nombre des cellules hépatiques représenté par l'acide désoxy-ribonucléique. Malgré le ralentissement de l'activité cellulaire indiqué par la chute de ce constituant, il y a accumulation de vitamine Brz dans le foie. L'étude de ces constituants cellulaires du foie n'infirme en rien les données de la première expérience quant à la vitamine Br2. Bien au contraire, elle renforce ces résultats et le pouvoir d'épargne des antibiotiques pour la vitamine BI2 est clairement mis en lumière.

Peut-on étendre ces conclusions aux autres vitamines du groupe B ? Ces vitamines n'ont pas figuré dans ce deuxième essai car le poids des foies des animaux n'a pas permis autant de dosages simultanés. Néanmoins, la confrontation des deux expériences révèle une double action de l'auréomycine. Elle augmente, d'une part, la teneur vitaminique du foie total et, d'autre part, elle abaisse notablement cette même teneur en acide désoxyribonucléique. On peut donc en conclure que le rapport :

\section{Vitamine du foie total \\ Acide désoxyribonucléique du foie total}

est très nettement amélioré sous l'effet de l'auréomycine et permet de mettre en évidence le rôle des antibiotiques comme facteurs d'épargne des vitamines $B$.

\section{Conclusions.}

L'auréomycine et, à un degré moindre, la pénicilline améliorent la croissance et 1 'indice de consommation de rats soumis à un régime subcarencé en vitamines $B$ (apportant le dixième des quantités optimales). Ces antibiotiques n'arrivent cependant pas à combler pleinement le retard du développement. 
Une telle action sur le gain de poids pourrait s'expliquer par le pouvoir d'épargne que jouent les antibiotiques pour les vitamines $B$, pouvoir qu'il est possible de démontrer directement au niveau du foie par l'étude de sa teneur relative en vitamines et en acides nucléiques. Ce rôle d'épargne est surtout manifeste pour la vitamine Br2. On peut donc admettre que les antibiotiques diminuent le besoin alimentaire en vitamines $B$, soit qu'ils favorisent le ravitaillement endogène par la flore intestinale, soit qu'ils réduisent les dépenses métaboliques.

On pourrait même aller plus avant et penser que cette théorie est également valable pour des facteurs de croissance encore indéterminés. Nous pensons ici aux " jus d'herbe " qui ont des propriétés nutritives spécifiques qu'on ne saurait attribuer à aucune vitamine identifiée. Ces propriétés se rapprochent de celles des antibiotiques en ce qui concerne la stimulation du développement. Or, en présence de " jus d'herbe " les antibiotiques perdent justement leur efficacité de croissance (I79 399).

CHAPI'TRE II

\section{INFLUENCE DES ANTIBIOTIQUES \\ SUR LA SYNTHÈSE MICROBIENNE DES VITAMINES B}

PAR

\section{CALET}

L'augmentation du rendement vitaminique sous l'effet des antibiotiques pose le problème suivant : l'organisme reçoit-il plus de vitamines ou bien les utilise-t-il mieux? Autrement dit, le pouvoir d'épargne des antibiotiques correspond-il à une diminution des besoins vitaminiques ou à une augmentation des vitamines synthétisées par la microflore de l'intestin?

Pour beaucoup d'auteurs, la stimulation de la croissance que provoquent les antibiotiques s'explique par des modifications de la flore intestinale. Depuis longtemps déjà, on connaissait l'importance de la flore intestinale (I47 - I87 - 250) pour l'état de santé générale. I1 existe une flore pathogène et une flore bénéfique. Plusieurs auteurs (37 - IIo - 244 - 246) ont souligné le bon effet de B. Bulgaricus dans le traitement des putréfactions intestinales. Par ailleurs, il est certain que 1'alimentation intervient directement sur la nature et la densité de la flore intestinale (I95 - 232 - 304-306-348-382$4 \mathrm{I} 7-4 \mathrm{I} 8)$. Certains auteurs ont donc pensé que les antibiotiques agissaient comme correcteurs d'une flore pathogène $(52-75-9 \mathrm{I}-$ $\mathrm{II} 8-\mathrm{I} 20-\mathrm{I} 2 \mathrm{I}-\mathrm{I} 24-\mathrm{I} 99-2 \mathrm{II}-2 \mathrm{I} 2-26 \mathrm{I}-4 \mathrm{I} 5)$. C'est la 
thèse d'ANDERSON, qui explique ainsi les effets favorables de la pénicilline pour le Poussin $(5-6)$.

Les antibiotiques agiraient, soit en éliminant les colonies prédatrices qui vivent en concurrence avec l'hôte (30), soit en favorisant les souches à grand pouvoir de synthèse vitaminique (I3), soit encore en orientant l'ensemble du métabolisme bactérien vers une plus grande élaboration de vitamines (I97 - 340). Dans les trois éventualités, il en résulterait une plus grande quantité de vitamines mises à la disposition de l'organisme et, par là-même, une protection dans les cas de carences alimentaires frustres.

De nombreuses études bactériologiques ont essayé de démontrer le bien fondé de cette théorie, que nous appellerons "théorie microbienne". Elles tendaient à caractériser les variations microbiennes sous l'effet des antibiotiques et sous le double aspect qualitatif (nature de la flore) et quantitatif (abondance de la flore).

Les études portaient sur les types microbiens les plus répandus : acidophiles, lactobacilles, coliformes, anaérobies et microaérophiles, aérobies, entérocoques, acétobacter, microcoques etc. (7 - II4 - I42 I65 - 349-376-388-389-438-440) et sur des formes plus rares : levures, sarcines, protei, clostridia $(65-23 I-340)$.

Certains auteurs ont soutenu que les antibiotiques inhibaient le développement de $C l$. perfringens $(295-340)$. Nous avons précédemment discuté de ce point. D'autres croient avoir enregistré une action positive des antibiotiques sur le développement des anaérobies et des coliformes et un effet négatif sur la croissance des entérocoques $(3-65-$ 308 - 395). Ces conclusions ont été contredites ultérieurement par leurs propres auteurs (I43 - 333). Par ailleurs, tous les autres essais donnent lieu à des résultats contradictoires.

Ainsi on a soutenu en même temps que le nombre des lactobacilles, forme très exigeante par ses besoins nutritionnels, était soit diminué $(23 \mathrm{I}-340-395)$ soit augmenté $(3-5)$, soit demeurait constant (I9 - I90) sous l'effet des antibiotiques. Ces faits étaient en liaison avec une modification du $\mathrm{pH}$ par les antibiotiques $(2-95)$. Enfin, un antibiotique donné, efficace pour la croissance, peut être favorable à une souche microbienne chez une espèce animale donnée et nuisible à la même souche chez une autre espèce animale. C'est le cas de $B$. coli dont la réponse aux antibiotiques est diamétralement opposée chez le Poulet et le Dindon (4 - 359).

Avec de nombreux auteurs (I43 - I44 - 3I5 - 374), on ne peut donc rien conclure de certain de ces seules études bactériologiques.

En sera-t-il de même pour les résultats des travaux métaboliques? Les études portaient alors sur le rôle des antibiotiques dans la synthèse bactérienne des vitamines $B$. D'une part, on a affirmé que les antibio- 
tiques modifiaient, dans le contenu intestinal (le caecum en particulier), le taux de thiamine (I89-44I), d'acide pantothénique $(\mathrm{I} 89-44 \mathrm{I})$, d'acide folique (307 - 353), de biotine (I97), et de vitamine BI2 (99I32 - I67).

D'autre part, tous ces faits ont été démentis $(90-378-379)$.

L'impression générale que laissent ces études est celle d'une telle variabilité des résultats d'un auteur à 1'autre que toute conclusion est impossible quant aux effets des antibiotiques sur la synthèse microbienne des vitamines $B$. Cela n'a rien de surprenant étant donné le caractère capricieux que nombre d'auteurs reconnaissent à la flore intestinale, irrégulière dans sa composition et inconstante dans son métabolisme.

Tel qu'il a été posé jusqu'ici, le problème des synthèses bactériennes manque de nuances. Du point de vue nutritionnel, il importe peu de savoir si la réfection des vitamines est le fait d'un changement de la flore ou d'une orientation nouvelle de son métabolisme. Par contre, il convient de savoir si les vitamines synthétisées sont utilisables par l'organisme. Quant au point précis d'un effet possible des antibiotiques, il faut donc établir une corrélation entre le bénéfice pondéral causé par l'antibiotique et le taux des synthèses utiles pour l'animal.

Le but de notre étude est donc de mettre en parallèle l'influence del'auréomycine sur la croissance du Rat, sur les réserves vitaminiques du foie et sur l'importance des synthèses microbiennes de quelques vitamines $B$.

Sur le plan biochimique, on peut aborder le problème en étudiant l'excrétion fécale des vitamines B et c'est ce que nous avons fait. Nous ne pouvions pas, en effet, poursuivre cette étude aux différents niveaux du tube digestif sans sacrifier l'animal. Or, il nous fallait garder les rats en vie pour apprécier les variations journalières de l'élimination fécale.

\section{I. - PREMIERE EXPERIENCE}

PAR

\section{CALET et A. RERAT}

ÉTUde INDIVIDUELLE, ANIMAI PAR ANIMAL.

\section{a) Conditions expérimentales.}

On étudie individuellement l'excrétion fécale de nicotinamide, riboflavine et vitamine BI2 chez des animaux soumis au régime de base suivant :

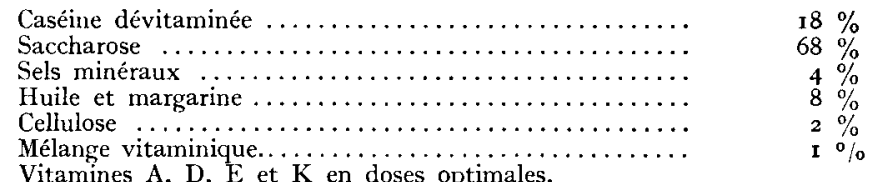




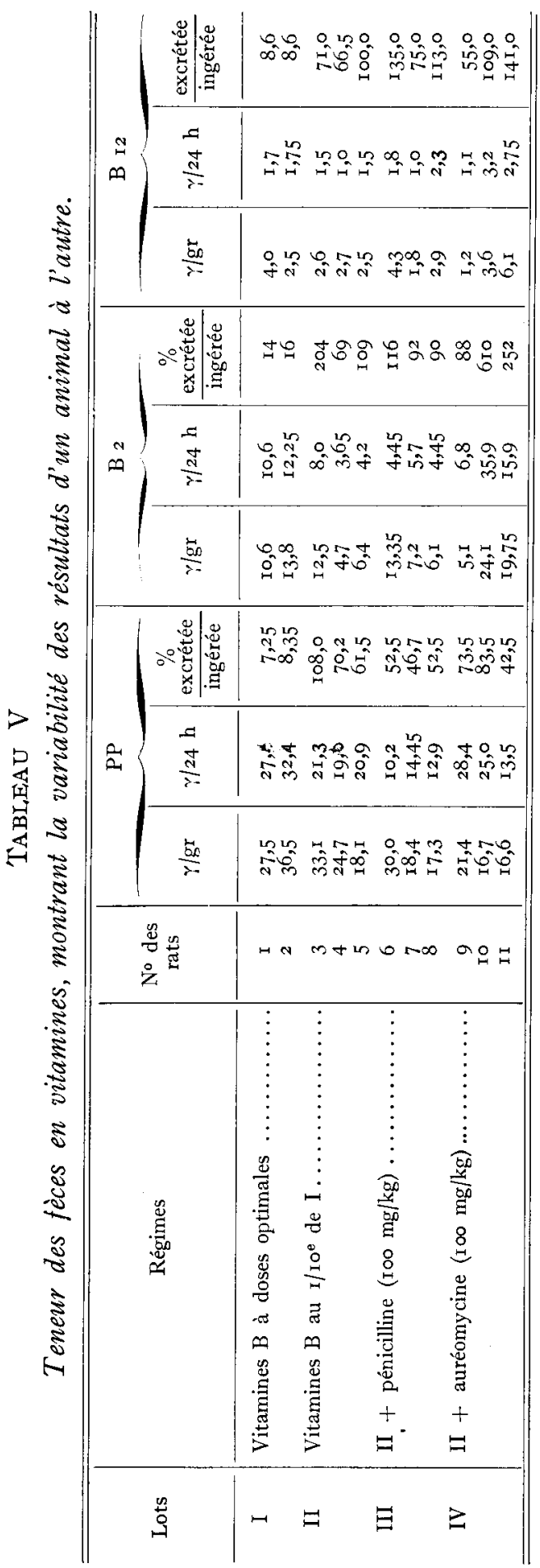

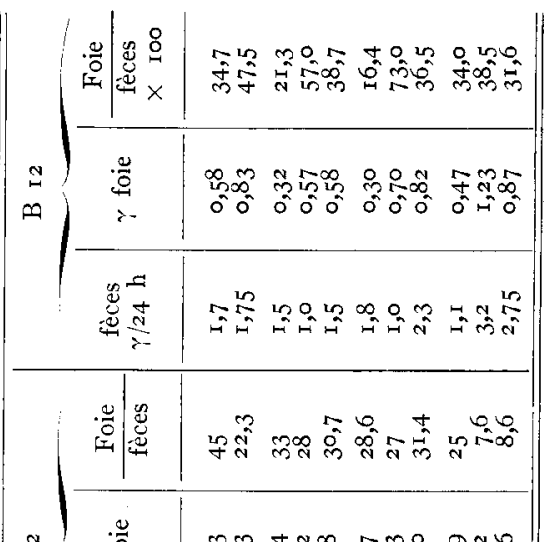

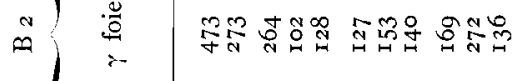

$\$$

3

1

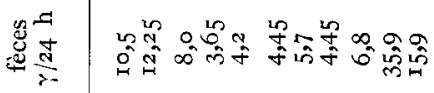

5
5
5
5
5
5
0
0
0

+4

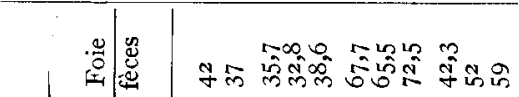

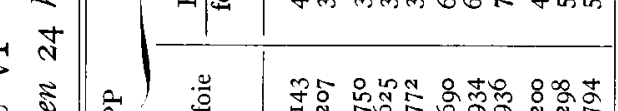

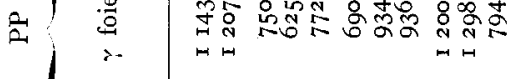

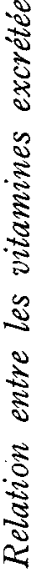

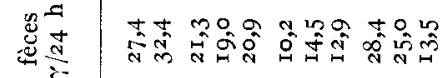

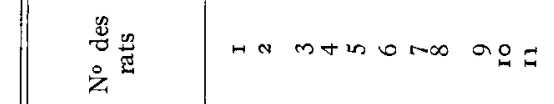

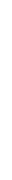

辛


Les rats sont répartis en quatre lots qui diffèrent soit par le taux de vitamines B ( $\left.{ }^{1}\right)$, soit par la présence et la nature de l'antibiotique. Les fèces sont récoltées deux fois par jour et stockées à $-5^{0}$ avant d'effectuer le dosage.

\section{b) Résultats expérimentaux.}

Le tableau $\mathrm{V}$ rapporte les résultats.

\section{Discussion des résultats}

A l'intérieur d'un même lot, les variations sont telles qu'elles rendent difficile l'interprétation des résultats. On peut néanmoins remarquer que :

- l'excrétion fécale des trois vitamines est du même ordre chez les animaux carencés et chez les sujets en régime équilibré ;

- les quantités de vitamines synthétisées par la flore peuvent donc être considérables : dans le cas de $\mathrm{BI}_{2}$, la synthèse microbienne atteint cent fois les taux ingérés;

- les antibiotiques n'exercent aucune influence particulière sur la teneur vitaminique des fèces.

Le tableau VI compare les taux d'excrétion par 24 heures et la teneur $\mathrm{du}$ foie en nicotinamide, riboflavine et vitamine BI2.

On ne voit à première vue aucune corrélation d'ensemble entre le taux des synthèses, représenté par l'excrétion fécale et la teneur hépatique en vitamines. Toutefois, le petit nombre des échantillons ne permettant pas le calcul statistique, aucune certitude ne peut être retirée de ces résultats.

\section{II. - DEUXIEME EXPÉRIENCE}

PAR

\section{CALET}

ÉTUdE CHRONOLOGIQUE, PÉRIODE PAR PÉRIODE.

\section{a) Conditions expérimentales.}

L'essai précédent portait sur le contenu vitaminique brut des fèces dans lequel on peut, a priori, distinguer deux fractions : une partie disponible et une partie inutilisable. La première semble correspondre aux formes solubles et facilement extractibles; la seconde serait représentée

(1) Le mélange vitaminique est celui décrit au Chap. I, page 22. Sa teneur en vitamines B diffère suivant les lots. 
par les vitamines de constitution des corps bactériens intacts. Il est bien connu, en effet que le caecum renferme en abondance des bactéries vivantes dont le contenu vitaminique est par là même "indisponible " pour l'hôte. En outre, les possibilités de résorption des vitamines sont fonction des caractères anatomiques et les lieux de synthèses peuvent être différents des lieux d'absorption.

\section{TABLEAU VII}

Composition de I $\mathrm{kg}$ des divers régimes.

\begin{tabular}{|c|c|c|c|c|}
\hline Régimes & 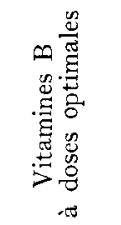 & 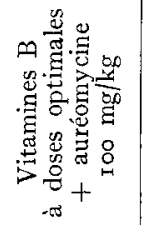 & 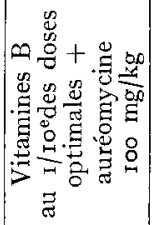 & 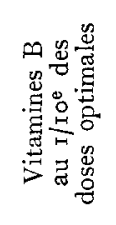 \\
\hline Lots & $\mathrm{SN}$ en $\mathrm{g}$ & $\mathrm{SAN}$ en $\mathrm{g}$ & SAC en $g$ & $\mathrm{SC}$ en $\mathrm{g}$ \\
\hline 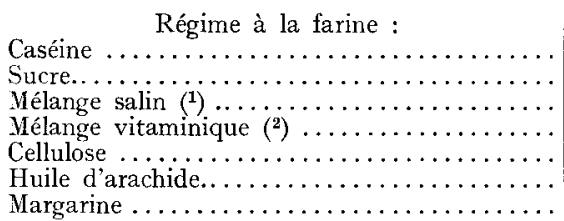 & $\begin{array}{r}200 \\
650 \\
40 \\
10 \\
20 \\
30 \\
50\end{array}$ & $\begin{array}{r}200 \\
650 \\
40 \\
10 \\
20 \\
30 \\
50\end{array}$ & $\begin{array}{r}200 \\
650 \\
40 \\
1 \\
20 \\
30 \\
50\end{array}$ & $\begin{array}{r}200 \\
650 \\
40 \\
1 \\
20 \\
30 \\
50\end{array}$ \\
\hline Lots & $\mathrm{FN}$ & FAN & $\mathrm{FAC}$ & $\mathrm{FC}$ \\
\hline 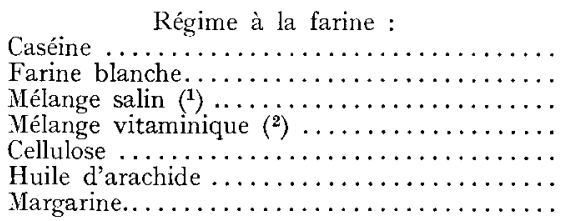 & $\begin{array}{r}100 \\
750 \\
40 \\
10 \\
20 \\
30 \\
50\end{array}$ & $\begin{array}{r}100 \\
750 \\
40 \\
10 \\
20 \\
30 \\
50\end{array}$ & $\begin{array}{r}100 \\
750 \\
40 \\
1 \\
20 \\
30 \\
50\end{array}$ & $\begin{array}{r}100 \\
750 \\
40 \\
1 \\
20 \\
30 \\
50\end{array}$ \\
\hline
\end{tabular}

(1) Mélange salin d'Osborne et Mendel, cf. Chapitre I. page 21.

(2) Mélange vitaminique normal (composition décrite au Chap. I page 22).

Dans cet essai, nous avons :

- d'une part, étudié l'élimination fécale des vitamines au cours de deux périodes successives;

- d'autre part, tenté de discriminer les formes vitaminiques disponibles des formes inutilisables;

- enfin fait varier la nature des glucides alimentaires qui conditionnent classiquement 1'intensité des synthèses.

Huit lots de six rats blancs pesant en moyenne $35 \mathrm{~g}$ au départ ont été nourris pendant ro semaines avec les régimes suivants, qui diffèrent notamment par la nature des glucides : farine blanche dans un cas dont 
l'amidon est très avantageux pour les processus de "réfection"; sucre dans l'autre, très peu favorable aux synthèses. Par ailleurs, les différences portaient comme précédemment sur le taux des vitamines $B$ et sur 1'adjonction d'antibiotique.

Le tableau VII résume ces dispositions.

Nota : Du point de vue des vitamines B, les lots des régimes au sucre ne sont pas comparables à ceux des régimes à la farine blanche. C'est en effet le même mélange vitaminique qui a été utilisé dans les deux cas, or la farine contient des vitamines B comme le montre le résultat des dosages effectués.

TABLEAU VIII

\begin{tabular}{|c|c|c|c|c|}
\hline & $\mathrm{B}_{1} \mathrm{mg}$ & $\mathrm{B}_{2} \mathrm{mg}$ & PP $\quad m g$ & $\mathrm{P} \mathrm{Ca} \mathrm{mg}$ \\
\hline 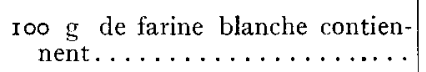 & $0,3 \mathrm{I}$ & 0,25 & $3,8 \mathrm{I}$ & 0,57 \\
\hline
\end{tabular}

Ce qui correspond dans les régimes carencés à la farine (FAC et $\mathrm{FC}$ ) à un apport vitaminique égal au $\mathrm{I} / 5^{\mathrm{e}}$ de celui des régimes normaux.

Les fèces sont récoltées plusieurs fois par jour et sont gardées à $-5^{\circ}$ jusqu'au moment du dosage. Pour éviter les grandes variations individuelles observées dans le premier essai, les récoltes d'un même lot sont réunies.

L'analyse vitaminique des fèces est conduite ainsi : les fèces fraîches sont broyées et mises en suspension dans du sérum physiologique, pour éviter la lyse des cellules vivantes. On centrifuge pendant $\mathrm{r} / 2$ heure à 5 ooo tours-minute, on décante et on recommence l'opération trois fois de suite en remettant chaque fois le culot en suspension dans du sérum physiologique. Les dosages microbiologiques se font sur l'ensemble des liquides surnageants (vitamines disponibles) et sur le culot (vitamines inutilisables). Pour chaque lot les résultats se groupent en deux périodes successives, ce qui permet d'apprécier les synthèses en fonction du temps.

\section{b) Résultats expérimentaux.}

I Le tableau IX rapporte les résultats d'ensemble.

On peut déduire de ces valeurs que :

- le taux vitaminique des fèces est plus élevé dans les lots placés aux régimes à base de farine que dans les lots au sucre; nous retrouvons un fait classique qui montre d'ailleurs que l'excrétion fécale renseigne utilement sur le taux des synthèses;

- l'auréomycine n'a pas d'effet propre sur l'intensité des synthèses ; 


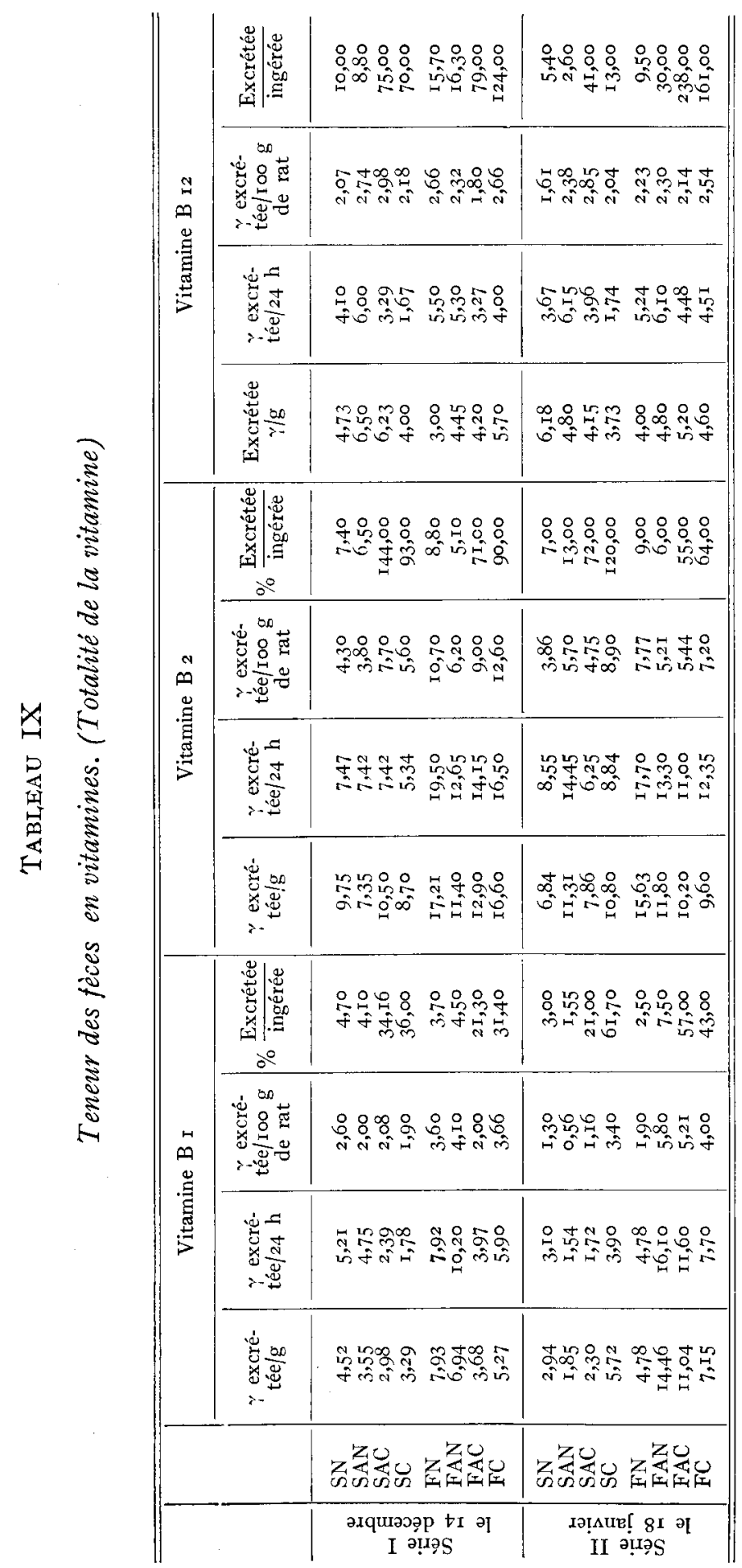




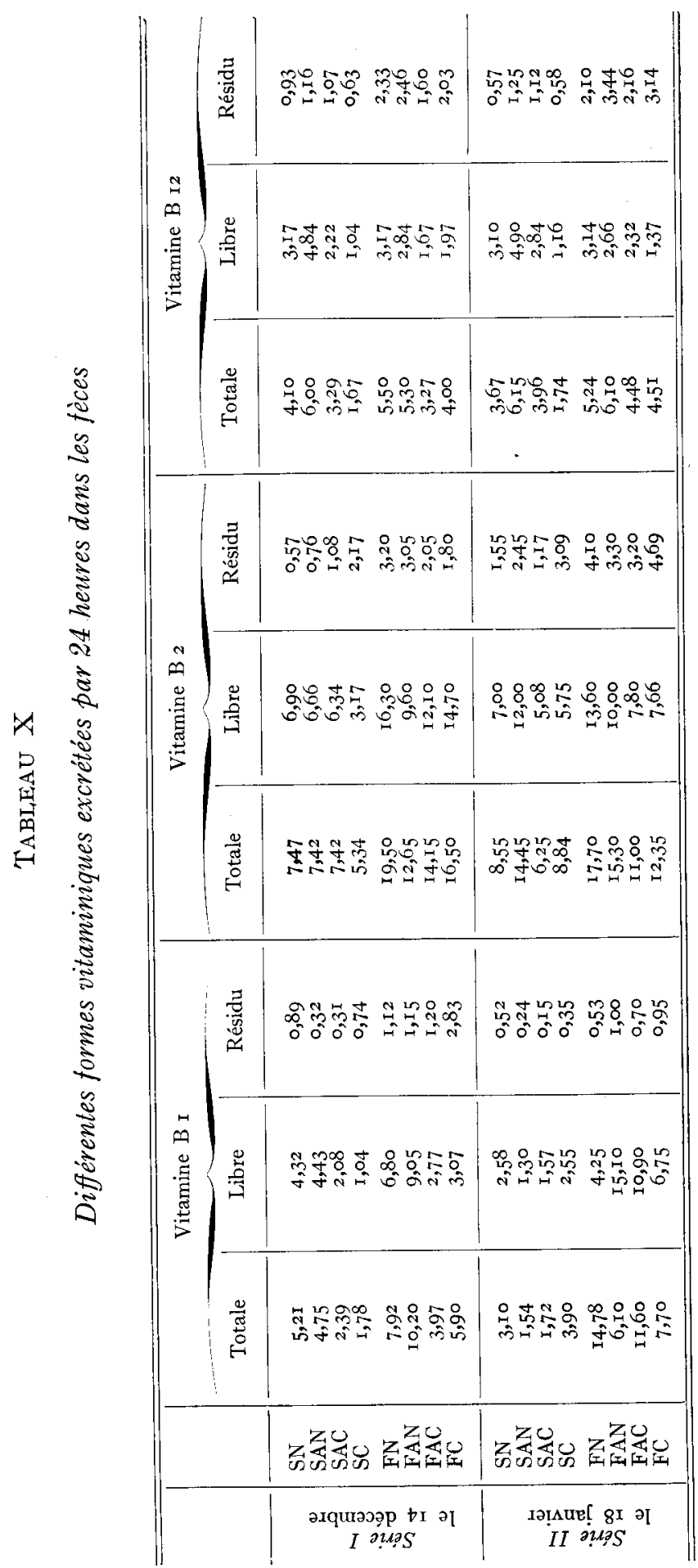


C. CALET

(I, I956)

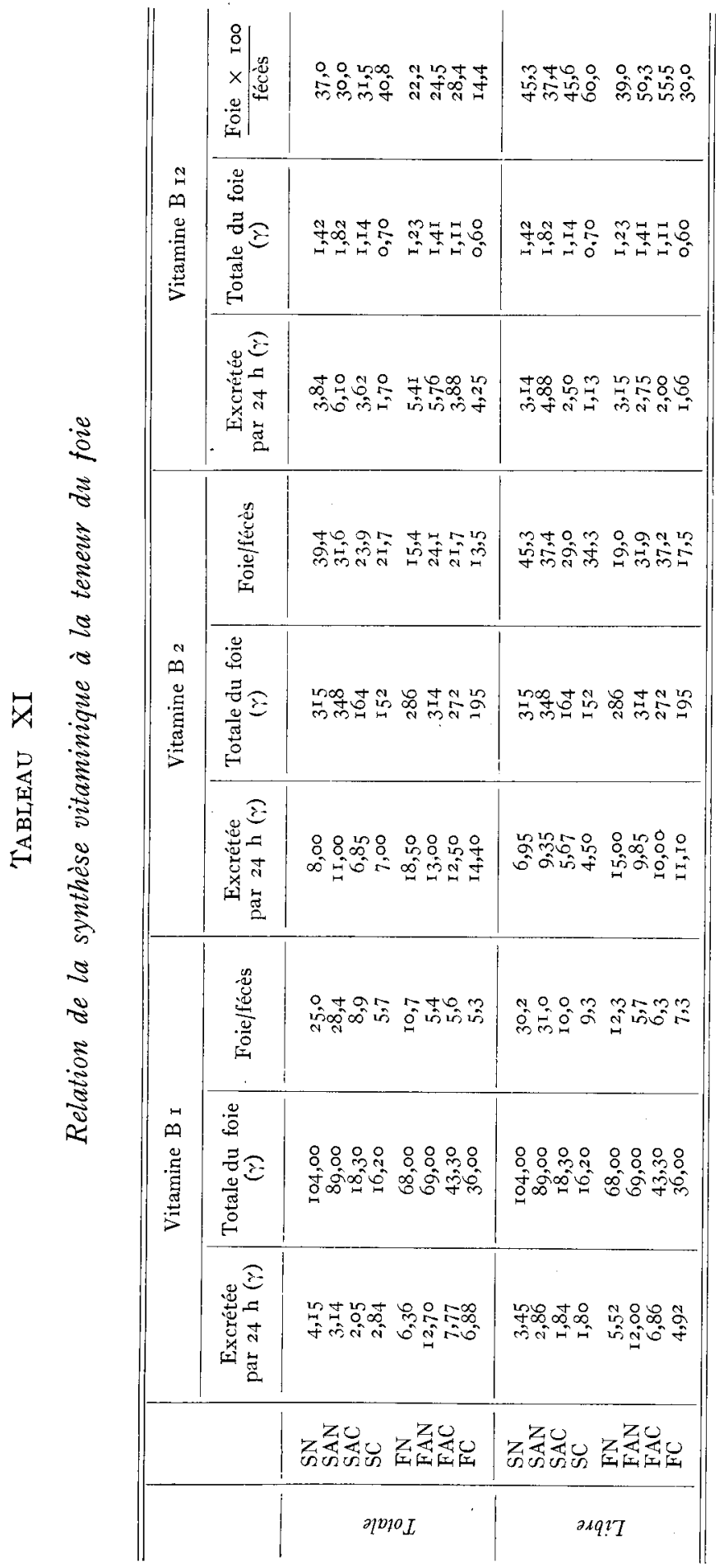


selon les périodes, elle diminue ou augmente le niveau d'excrétion par rapport aux témoins.

$2^{0}$ Si l'on considère la répartition des différentes formes de vitamines, le tableau $\mathrm{X}$ nous renseigne comme suit :

- il y a toujours dans les fèces plus de vitamines disponibles que de vitamines inutilisables;

- l'auréomycine n'influence pas la répartition entre les deux fractions.

$3^{\circ}$ Comme dans la première expérience, nous avons comparé les quantités de vitamines excrétées dans les fèces à celles retenues dans le foie.

Ici aussi le tableau XI ne peut mettre en évidence la moindre relation entre intensité des synthèses et teneur du foie en vitamines. Le fait est frappant si l'on considère que, dans chaque série homologue, les rats alimentés à la farine n'ont pas de réserves vitaminiques plus élevées, bien que leur niveau d'excrétion soit nettement plus haut.

D'une manière générale, nous ne voyons donc aucun lien entre intensité des synthèses microbiennes dans le tube digestif et grandeur des réserves hépatiques. L'exemple de $\mathrm{Br} 2$ est significatif ; on note une synthèse énorme de vitamine " disponible " et cependant la teneur des foies est inchangée. Cela veut-il dire que la portion du tube digestif où les vitamines peuvent être absorbées est très limitée et se place à un niveau anatomiquement plus élevé que les foyers de synthèse?

Comme d'autre part rien ne permet d'affirmer que l'auréomycine intervient dans l'élaboration bactérienne des vitamines $B$, on peut en déduire que l'action de cet antibiotique sur la croissance et son pouvoir d'épargne vis-à-vis des vitamines $B$ ne relève pas directement d'une activité accrue de la flore intestinale dans les processus de vitaminogenèse.

Nos conclusions concordent donc avec celles des bactériologistes. La bibliographie regorge de données contradictoires sur la nature et le nombre des microorganismes qui se développent dans l'intestin en présence d'antibiotique. Il est bien évident, si les souches microbiennes sont si changeantes, que le résultat de leur métabolisme le soit aussi.

Malgré le manque de relations entre l'intensité des réfections vitaminiques et le taux des réserves hépatiques, la nature des glucides alimentaires conditionne cependant la valeur nutritive des régimes subcarencés en vitamines $\mathrm{B}$.

\section{c) Observations et mesures sur animaux.}

L'aspect général des animaux est bien meilleur dans les lots alimentés à la farine que dans les lots nourris au sucre. Dans le premier 
cas, la privation de vitamines $B$ 'n'altère pas la fourrure qui reste abondante et soyeuse alors que les animaux soumis au régime dévitaminé sucré accusent, dès la cinquième semaine, des signes carentiels épidermiques se traduisant par l'alopécie et des dépôts de porphyrine sur les poils. I'auréomycine, dans ce cas, loin de prévenir l'apparition des signes carentiels, semble les accuser. Le seul effet favorable de l'antibiotique est de diminuer l'incidence des diarrhées et le taux de mortalité.

Le tableau XII groupe l'ensemble des observations in vivo.

\section{TABLEAU XII}

\begin{tabular}{|c|c|c|c|c|c|c|c|c|}
\hline Régimes & 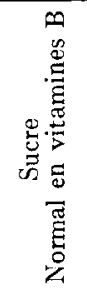 & 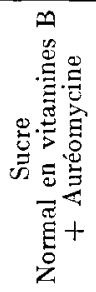 & 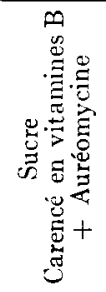 & 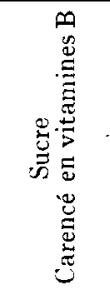 & 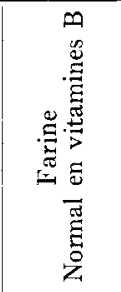 & 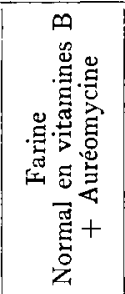 & 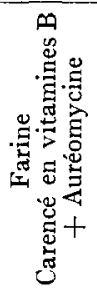 & 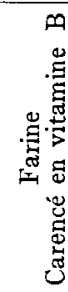 \\
\hline $\begin{array}{l}\text { Lots .......... } \\
\text { Mortalité } \%\end{array}$ & $\begin{array}{c}\text { SN } \\
0\end{array}$ & $\underset{\circ}{\text { SAN }}$ & $\begin{array}{c}\mathrm{SAC} \\
0\end{array}$ & $\begin{array}{c}\mathrm{SC} \\
20 \%\end{array}$ & $\begin{array}{c}\text { FN } \\
0\end{array}$ & $\underset{\circ}{\text { FAN }}$ & $\underset{0}{\text { FAC }}$ & $\begin{array}{c}\text { FC } \\
0\end{array}$ \\
\hline $\begin{array}{l}\text { Etat général en } \\
\text { fin d'expé- } \\
\text { rience } \ldots . . \\
\text { Gain de poids }\end{array}$ & Bon & Bon & $\begin{array}{c}\text { Très } \\
\text { mauvais }\end{array}$ & mauvais & Excellent & Excellent & Bon & Bon \\
\hline $\begin{array}{c}\text { total (g) } \\
\text { Nourriture } \\
\text { in- }\end{array}$ & 182 & $2 \perp 2,5$ & 95 & 62 & 190 & 212 & I 63,5 & 135 \\
\hline $\begin{array}{l}\text { gérée (g) .... } \\
\text { Indice de con- }\end{array}$ & 812 & 864 & 542 & $47^{6}$ & 780 & 865 & 7.36 & 680 \\
\hline sommation ... & 4,45 & 4,02 & $5,7^{\circ}$ & 7,70 & 4,08 & 4,08 & 4,5 & $5, \mathrm{I}$ \\
\hline
\end{tabular}

Du point de vue de la croissance, les lots normaux accusent le même développement pondéral, qu'ils soient alimentés au sucre ou à la farine. L'addition d'auréomycine est quand même efficace. Au contraire, le régime sucré et carencé est nettement moins favorable à la croissance que le régime carencé à la farine. En réalité les lots ne sont pas comparables, la farine apportant une petite quantité de vitamines que le sucre ne contient pas. L'addition d'auréomycine est efficace dans les deux cas, mais l'efficacité est d'autant plus marquée que le régime est moins apte (régime au sucre).

En résumé, dans ces expériences, l'effet de croissance de l'auréomycine peut ainsi se traduire sous l'angle des augmentations de gain de poids :

- I7 p. Ioo avec le régime normal au sucre.

- 53 p. Ioo avec le régime carencé au sucre.

- II p. Ioo avec le régime normal à la farine.

- 2 I p. Ioo avec le régime carencé à la farine. 


\section{Conclusions}

Nos observations ne permettent aucunement de mettre en évidence l'influence de l'auréomycine sur le taux des synthèses microbiennes de vitamines $\mathrm{B}$ ou sur la forme des vitamines synthétisées (utilisables ou non par l'animal). Par ailleurs, nous n'avons trouvé aucune corrélation entre l'intensité de la croissance des rats et la teneur vitaminique de leurs fèces.

Ces résultats négatifs sont à rapprocher des études déjà anciennes faites sur les sulfamides $(49-25 \mathrm{I})$. Ici, aussi, et par recoupement entre les auteurs, on voit que ces médicaments, administrés per os, stimulent la croissance des animaux et modifient leur flore, sans augmenter cependant l'importance des synthèses intestinales ( $147-3 \mathrm{I} 4-380)$. Bien mieux, les sulfamides peuvent parfois créer des carences vitaminiques par destruction de la flore (I27 - I29 - I97). En plus, l'effet des sulfamides sur la nature et l'abondance de la flore n'est pas plus reproductible que l'effet des antibiotiques.

En réalité, la flore est directement placée sous 1'influence de 1'alimentation. Tous les principes alimentaires peuvent la modifier. Il peut s'agir d'un effet indirect comme celui des protides et des lipides qui sélectionnent la flore en abaissant le $\mathrm{pH}(4-359)$. Il peut s'agir d'un effet direct comme celui des glucides qui fournissent un aliment de choix pour la synthèse des vitamines $B(147-187-250)$. Nos propres résultats viennent confirmer ceux des auteurs précédents qui estiment que la prolifération des bactéries et la synthèse microbienne des vitamines B dépendent essentiellement du taux et de la nature des glucides alimentaires, qu'il y ait antibiotiques ou non (42I).

En plus du rôle propre des différents principes alimentaires, on a voulu invoquer le facteur appétit. Tous les auteurs soulignent que les antibiotiques améliorent l'appétence et font que l'animal mange plus $(62-7 \mathrm{I}-8 \mathrm{I}-24 \mathrm{I})$. On a même prétendu que les antibiotiques n'étaient plus efficaces avec des régimes limités $(384)$. Certains auteurs ont dès lors établi une relation de cause à effet entre l'influence des antibiotiques sur l'appétit et le nombre de coliformes (395).

Dans le même ordre d'idée, BRIDGes relie l'action des antibiotiques sur la teneur en $B$. coli du tube digestif au gain de poids (65). Cependant des études toutes aussi sérieuses démontrent que les antibiotiques seuls n'ont aucun effet sur la prolifération des formes coli, qui dépend uniquement de la nature des sucres de la ration (444).

Même s'ils avaient été positifs, nos résultats demanderaient à être discutés avant d'affirmer que les antibiotiques agissent sur la croissance par le relai de la flore intestinale. Dans l'introduction, nous avons déjà 
mentionné les réserves qu'appelle une telle conception. Les arguments suivants la rendent encore plus précaire.

I $^{0}$ Si l'efficacité des antibiotiques était liée au développement de formes microbiennes particulières, l'ingestion d'une culture de ces bacilles devrait reproduire le même effet (349).

L'essai a été tenté (5-6), mais s'est révélé incertain. On a bien signalé dans ces conditions une faible réponse de la croissance, mais 1'adjonction d'antibiotiques à la culture augmentait considérablement l'effet produit.

$2^{\circ}$ A elle seule l'auréomycine suffit à augmenter la teneur en vitamine BI2 du caecum et du colon ; pourtant la croissance est meilleure si la ration contient en plus de la vitamine $\mathrm{BI} 2$ (3I9). Si la ration est déficiente en BI2 la teneur des foies et des reins en cette vitamine n'est pas rehaussée par l'auréomycine : utilisant la radiocobalamine CHOw (99 - r32) coordonne ces résultats en apparence disparates : 1'auréomycine multiplie par 8 la teneur des fèces en Br2, mais le foie n'en retire aucun bénéfice. Les études sur Porc arrivent aux mêmes conclusions ( I9 - 90). On peut dès lors penser que la vitamine BI2 synthétisée n'est pas utilisable, soit en raison du lieu des synthèses et d'un défaut d'absorption (306), soit qu'il s'agisse de cobalamines sans action vitaminique ( 167 ) (on a décrit en effet des pseudovitamines BI2 dans les excréments (I06 - I68)).

$3^{\circ}$ On sait que les antibiotiques agissent sur la flore du rumen ; pourtant leur emploi se montre défavorable chez les ruminants adultes (245$359-365)$.

$4^{\circ}$ Un argument de poids en faveur de 1'action directe des antibiotiques sur la flore intestinale réside dans le fait qu'ils sont dépourvus d'efficacité chez les animaux élevés stérilement $\left(27^{6}-350-35 \mathrm{I}-35^{2}\right)$. On en déduit que n'ayant pas deflore intestinale, ces animaux ne peuvent bénéficier de la réfection vitaminique.

Cette notion implique qu'en cas de carence, toutes les vitamines des fèces sont d'origine microbienne. Or, L,UCKEY (278) dans une récente expérience vient de montrer que des poussins stériles et rigoureusement carencés en vitamines $B$ excrètent cependant des quantités notables de ces vitamines par les fèces. Cet auteur en déduit que l'action de la microflore intestinale est surestimée dans le mécanisme d'action des antibiotiques.

$5^{\circ}$ L'action des antibiotiques sur la synthèse intestinale de formes vitaminiques assimilables serait bien établie si la teneur en vitamine $B$ de la veine porte était accrue par le traitement. Une telle expérience chez le veau (402) aboutit à un échec : 1'auréomycine ne modifie en rien la concentration sanguine en thiamine, riboflavine, nicotinamide, acide pantothénique et vitamine $\mathrm{BI} 2$.

En résumé, on ne peut établir aucune corrélation entre l'effet d'épargne de l'auréomycine vis-à-vis des vitamines $B$ et l'intensité des synthèses intestinales appréciées par le taux vitaminique des fèces. Dans 
nos essais, l'auréomycine ne favorise pas ces synthèses. Ce qui conduit à penser que tous les animaux carencés disposent des mêmes quantités de vitamines au niveau de l'absorption intestinale, qu'ils reçoivent ou non des antibiotiques. Autrement dit, l'effet de croissance de l'auréomycine et son pouvoir d'épargne pour les vitamines $B$ ne comporteraient pas un relai obligatoire par la flore intestinale, mais relèveraient d'un véritable processus métabolique au niveau des tissus.

\section{CHAPITRE III}

\section{SIGNIFICATION DES RELATIONS ENTRE VITAMINE B12 ET AUREOMYCINE}

Le pouvoir d'épargne de l'auréomycine pour la vitamine BI2 vient d'être mis en évidence. Il s'agit d'un processus métabolique qui pourrait impliquer une similitude d'action entre la vitamine et l'antibiotique. En poussant les choses à l'extrême, on pourrait penser à des phénomènes de " vicariance ", les deux substances étant interchangeables quant à leur effet métabolique. Cette idée est d'autant plus présente à l'esprit que, dans la pratique, vitamine $\mathrm{BI} 2$ et auréomycine sont souvent confondues dans le même vocable d' "Animal Protein Factor ". Les spécialités vendues sous ce nom correspondent à des sous-produits riches à la fois en cyanocobalamine et en auréomycine.

Le problème posé est de savoir si le pouvoir d'épargne pour la vitamine BI2 peut rendre compte à lui seul de l'action favorable des antibiotiques sur la croissance. Cette hypothèse impliquerait deux corollaires, à savoir la complète interchangeabilité de l'auréomycine et de BI2 dans le régime, la généralité du rôle de $\mathrm{BI} 2$ comme facteur de croissance. Pour les vérifier, nous avons réalisé les essais suivants.

\section{I. - INTERCHANGEABILITÉ « VITAMINE B12 - A URÉOMYCINE » PAR}

\section{CALET et A. RERAT ( $\left.{ }^{1}\right)$}

Pendant neuf semaines, 5 lots de 9 rats pesant $37 \mathrm{~g}$ au départ sont soumis au régime de base suivant :
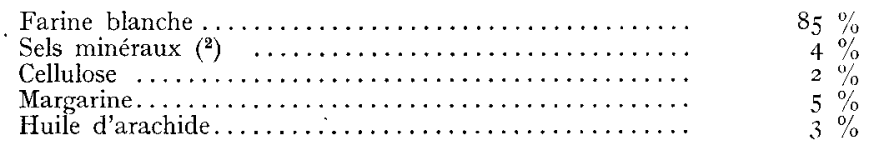

(1) Avec la collaboration technique de Mmes de Saintaurin et Macaire.

(2) Mélange salin de Osborne et Mendel (voir chap. I, page 2I). 
La répartition des lots est la suivante :

Lot I. - R.B. + Mélange vitaminique ( $\left.{ }^{(}\right)$I $\%$.

Lot II. - R.B. + Mélange vitaminique I $\%+\mathrm{BI2}(30 \gamma / \mathrm{kg})$.

Lot III. - R.B. + Mélange vitaminique I \% + auréomycine (Ioo mg/

$\mathrm{kg})$.

Lot IV. - R.B. + Mélange vitaminique I $\%$ + $\mathrm{BI} 2$ + auréomycine.

Lot V. - R.B. + BI2 (30\%/kg).

Ainsi, pour les quatre premiers lots, on utilise toutes les combinaisons possibles entre $\mathrm{BI} 2$ et auréomycine. Le lot $\mathrm{V}$ ne contient que les très faibles quantités de vitamines B apportées par la farine ; il est, par contre, enrichi en vitamine $\mathrm{BI} 2$. I1 a été choisi pour tenter de voir si la vitamine $\mathrm{Br}$, comme l'auréomycine, exerce une action d'épargne sur les autres vitamines $B$.

\section{RÉSULTATS EXPÉRIMENTAUX}

\section{a) Aspect des animaux.}

Les animaux restent petits mais sont assez vifs en général, sauf pour le lot V. Les animaux de ce lot sont en effet les premiers à manifester les signes de carence : dépôt de porphyrine sur les poils, yeux gonflés et injectés de sang, eczéma suintant sur les muqueuses, ventre ballonné et diarrhées. Souvent les poils se collent en mèches et tombent. Ces animaux résistent très mal aux parasites.

Ces mêmes signes apparaissent dans les autres lots plus ou moins tôt et avec plus ou moins d'intensité. Toutefois, ce sont les rats qui reçoivent de 1'auréomycine qui présentent le plus mauvais aspect : ou bien l'animal n'a plus de poils, ou bien ceux-ci sont recouverts d'épais dépôts de porphyrine. Cependant, dans ces lots, on ne note pas de troubles intestinaux apparents.

b) Le tableau XIII rapporte les résultats moyens relatifs à la croissance et à l'indice de consommation.

Ces résultats se répartissent avec une grande netteté en deux groupes : - seuls les lots qui reçoivent de l'auréomycine (lots III et IV) lange) :

(1) Ce mélange vitaminique privé de $\mathrm{B}$ r 2 a la composition suivante (exprimée en g par kg de mé-

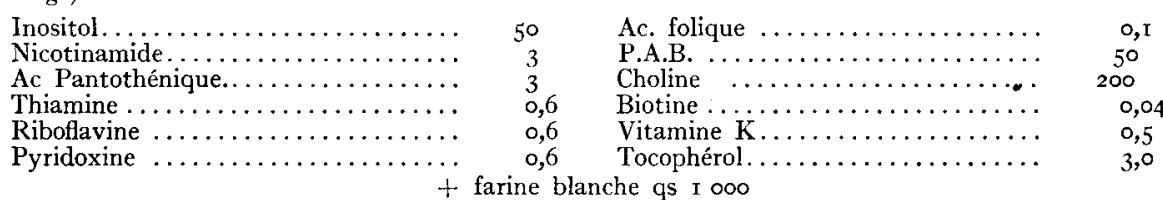

Les vitamines $\mathrm{A}$ et $\mathrm{D}$ sont apportées per os en solution huileuse à raison de zo $000 \mathrm{U}$. I. de $\mathrm{A}$ et 2500 U. I. de D par semaine. 
TABleau XIII

\begin{tabular}{|c|c|c|c|c|c|}
\hline Lots & $\underset{9 \text { rats }}{\text { I }}$ & $\begin{array}{l}\text { II } \\
9 \text { rats }\end{array}$ & $\begin{array}{l}\text { III } \\
9 \text { rats }\end{array}$ & $\begin{array}{l}\text { IV } \\
9 \text { rats }\end{array}$ & $\begin{array}{l}\mathrm{V} \\
9 \text { rats }\end{array}$ \\
\hline $\begin{array}{l}\text { Poids final }(\mathrm{g}) \ldots \ldots \ldots \ldots \ldots \\
\text { Gain de poids total }(\mathrm{g}) \ldots \ldots \ldots \ldots \ldots \\
\text { Ecart-type du gain de poids...... } \\
\text { Nourriture ingérée }(\mathrm{g}) \ldots \ldots \ldots \ldots \ldots \\
\text { Ecart-type de la nourriture ingérée. } \\
\text { Indice de cunsommation. . . . . . . . }\end{array}$ & $\begin{array}{r}61,5 \\
24,5 \\
8,5 \\
308 \\
30,2 \\
14,4\end{array}$ & $\begin{array}{r}59,2 \\
23,4 \\
6,8 \\
301 \\
32,5 \\
14,5\end{array}$ & $\begin{array}{c}72,3 \\
35,3 \\
7,25 \\
342 \\
41,2 \\
9,9\end{array}$ & $\begin{array}{r}7 \pm, 4 \\
34,5 \\
7,5 \\
33^{6} \\
49,3 \\
0,9\end{array}$ & $\begin{array}{l}56,2 \\
17,0 \\
4,75 \\
300 \\
16,3 \\
18,8\end{array}$ \\
\hline
\end{tabular}

accusent un développement supérieur à celui des témoins (lot I) ; la vitamine Bız n'a, par elle-même, aucun effet de croissance. L'auréomycine améliore de $47 \mathrm{p}$. Ioo le gain de poids et l'indice de consommation. Le calcul statistique montre que cette différence est hautement significative. Notons cependant que les quantités de nourriture ingérée, sans que ce résultat soit significatif, sont toujours plus élevées chez les animaux les plus gros.

Ces résultats démontrent donc que, avec ou sans auréomycine, l'action de la vitamine $\mathrm{BI} 2$ dans notre essai est rigoureusement nulle sur la croissance. Les résultats donnés par le lot $\mathrm{V}$ prouvent que, à elle seule, la vitamine BIz ne permet pas de compenser le retard provoqué par la carence des autres vitamines $B$.

\section{c) Étude de la teneur vitaminique des foies.}

Au bout de 9 semaines les animaux sont sacrifiés et les foies sont prélevés. Enn dehors du lot $\mathrm{V}$ où les foies sont nettement gros du fait de la carence vitaminique $B$, les foies des animaux des autres lots sont normaux. Après avoir stocké les foies à - $20^{\circ}$ jusqu'au moment de leur étude biochimique, les dosages de $\mathrm{BI} 2$ ont donné les résultats suivants :

TABLEAU XIV

\begin{tabular}{|c|c|c|c|c|c|}
\hline \multirow{2}{*}{$\frac{\text { Régimes }}{\text { Lots }}$} & \multicolumn{2}{|c|}{ Sans B 12} & \multicolumn{3}{|c|}{ Avec B I 2} \\
\hline & I & III & II & IV & V \\
\hline Poids du foie frais $(g) \ldots \ldots \ldots \ldots$ & $3, \mathrm{I} 5$ & 3,3 & 2,7 & 3,2 & 3,7 \\
\hline 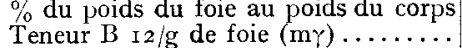 & $\begin{array}{r}5,0 \\
\text { I02,7 }\end{array}$ & $\begin{array}{r}4,5 \\
73,2\end{array}$ & $\begin{array}{r}4,6 \\
337,7\end{array}$ & $\begin{array}{r}4,6 \\
25 \mathrm{I}, \mathrm{o}\end{array}$ & $\begin{array}{r}6,4 \\
300,0\end{array}$ \\
\hline $\begin{array}{l}\text { Teneur totale du foie en } \mathrm{B} \text { I } 2(\mathrm{~m} \gamma) \text {. } \\
\text { B I } 2 \text { totale du foie }(\mathrm{m} \gamma) \text { calculée par }\end{array}$ & 302,5 & $240, \mathrm{I}$ & $905, \circ$ & 848,0 & I 096,0 \\
\hline $\begin{array}{l}\text { Ioo } \mathrm{g} \text { de rat } \ldots \ldots \ldots \ldots \ldots \ldots \ldots \ldots \\
\text { Gain de poids }(\mathrm{g}) \ldots \ldots \ldots \ldots \ldots \ldots \ldots \ldots\end{array}$ & $\begin{array}{r}497,5 \\
24,5\end{array}$ & $\begin{array}{r}337,5 \\
72,3\end{array}$ & $\begin{array}{r}\text { I } 742,0 \\
59,2\end{array}$ & $\begin{aligned} \text { I } 332,0 \\
7 \mathrm{r}, 4\end{aligned}$ & $\begin{array}{l}\text { I } 921,0 \\
\text { I } 7,0\end{array}$ \\
\hline
\end{tabular}

Nous avons là des résultats extrêmement nets quant à la composition du foie. On peut à nouveau répartir les animaux en deux groupes : ceux 
qui ingèrent de la vitamine BI2 et ceux qui n'en reçoivent pas. Toutefois, cette répartition ne correspond nullement à la précédente : qu'il y ait auréomycine ou non, le stockage de $\mathrm{BI} 2$ dans le foie est uniquement le reflet de la vitamine ingérée. Ramenée à 1'unité du poids vif, la vitamine BI2 hépatique est quatre fois plus importante quand la ration en contient. Enfin, quel que soit le mode d'expression des résultats employés, il n'y a aucune relation entre la vitamine BI2 du foie et le gain de poids. Le lot V en apporte une démonstration flagrante : il est constitué d'animaux dont le taux de croissance est le plus faible et où le stock de BI2 du foie est le plus élevé.

Non seulement 1'auréomycine n'augmente pas le taux hépatique de vitamine $\mathrm{BI} 2$, mais elle semble même le diminuer légèrement comme le montre la comparaison entre les lots II et IV d'une part, les lots I et III d'autre part.

\section{Discussion}

Nos résultats doivent être commentés sur un double plan : croissance et réserves vitaminiques.

Io Dans cet essai, le lot soumis au mélange " auréomycine-BI2 " n'accuse pas un gain de poids supérieur à celui du lot recevant l'auréomycine seule. L'auréomycine valorise 1'efficacité pour la croissance d'un régime à base de farine blanche alors que la vitamine $\mathrm{Br} 2$ est sans action.

Cette conclusion va de pair avec certaines observations antérieures $(77-335-355-392)$. Rares sont les résultats où les antibiotiques et la vitamine BI2 ont des effets similaires.

Le mélange " antibiotique-BI2 " est plus efficace que la seule vitamine pour la croissance du Porc (I40 - 280), du Poulet (233) et du Rat (43 - 239-4I3). JukEs et col. (239) d'une part, CoAtes et col. (I05) de l'autre, n'ont jamais pu reproduire l'effet des antibiotiques par surcharge du régime en vitamine BI2 à quelque dose que ce soit.

La terramycine favorise la ponte, augmente le poids des poussins nouveaux-nés et relève le taux d'éclosabilité sans pour cela accroître la teneur du jaune d'œuf en vitamine BI2 (29I). Il en est de même de la streptomycine qui n'agit pas par 1'intermédiaire de la vitamine BI2.

Cependant, d'autres auteurs ont une opinion différente (I82). Analysant les rôles relatifs de la streptomycine et de la vitamine BI2 dans l'effet de croissance, BLACK et BRATZLER attribuent la part la plus importante à la vitamine (48). On a également souligné que les antibiotiques étaient inopérants à améliorer la croissance de poussins issus de mères carencées en BI2 (40 - I04 - I07 - I96-234-253-455). Bien que ces faits semblent contradictoires, ils laissent déjà supposer une 
différence foncière dans le mécanisme d'action des antibiotiques et de la vitamine $\mathrm{BI}$.

$2^{\circ}$ Dans notre essai, le taux des réserves hépatiques en vitamine BI2 est indépendant de l'adjonction d'auréomycine à la ration et de 1'abondance des autres vitamines $B$. Il dépend uniquement des quantités de vitamine $\mathrm{BI} 2$ ingérée.

Cette conclusion a été confirmée par de nouvelles observations : le taux des vitamines $\mathrm{BI} 2$ hépatique et rénal est entièrement fonction de l'administration de cette vitamine au Poussin (II5 - I43) et au Porc, comme cela a été confirmé ultérieurement à nos propres résultats (I9 I63).

Les bilans montrent ce stockage tissulaire puisque $6 \mathrm{p}$. Ioo seulement de la vitamine injectée se retrouve dans l'urine (24). Le même résultat a été reproduit chez l'homme en utilisant la radiocobalamine (IOO).

Dans tous les cas, il n'y a pas de relation entre le stockage hépatique de la vitamine et l'intensité de la croissance (r30 - $\mathrm{r}_{36}-256-257-$ $299-3$ I0).

D'autres résultats montrent également l'indépendance des effets de la vitamine $\mathrm{Br} 2$ et des antibiotiques :

- les antibiotiques ne remédient pas aux troubles caractéristiques de $\mathrm{BI} 2$ tels que les hémorragies rénales (210 - 375- 464), l'élévation de l'urée sanguine $(206-296-38 \mathrm{I}-465)$, la chute de l'hémoglobine et la diminution des globules rouges $(\mathrm{I} 2-\mathrm{I} 69)$;

- la pénicilline ne modifie pas le besoin en $\mathrm{BI} 2$ nécessaire à la bonne éclosion des œufs (I82).

Tous ces faits épaulent donc nos propres résultats qui se résument en une double notion:

- malgré son pouvoir d'épargne qui se manifeste pour les faibles doses de $\mathrm{BI}$, l'auréomycine ne saurait nullement se substituer à la vitamine pour la constitution des réserves hépatiques;

- du point de vue efficacité pour la croissance, l'auréomycine présente un effet que ne partage aucunement la vitamine BI2.

Il ne saurait donc y avoir interchangeabilité entre les deux substances. 


\section{II. - ROLE DE LA VITAMINE BI2 COMME FACTEUR DE CROISSANCE}

PAR

\section{CALET}

Dans l'essai précédent, la vitamine Bı2 n'a aucune efficacité pour la croissance. Ce résultat est en désaccord avec la notion que cette vitamine, constituant essentiel du "Facteur des Protéines Animales" (A.P.F.), valorise à coup sûr tous les régimes à base de protéines végétales. En fait, i1 semble que l'efficacité pour la croissance de la vitamine Br2 soit fonction de l'équilibre des aminoacides du régime. C'est pourquoi nous avons choisi des types de rations bien différents quant à la nature du facteur limitant, méthionine dans un cas, lysine dans 1'autre. Pour aller plus loin et tenter de démontrer le lien éventuel entre Brz et croissance, nous avons pris la réserve hépatique comme test d'efficacité vitaminique.

Enfin, comme on connaît l'importance des synthèses de BI2 dans le tube digestif et pour tenir compte de cette source éventuelle d'origine endogène, nous avons utilisé comparativement deux types de glucides : amidon et sucre. Le premier permet en effet des synthèses intestinales importantes, alors que le sucre entraîne une réfection beaucoup plus faible.

L'essai que nous rapportons maintenant porte sur 6 lots de 8 rats blancs maintenus aux régimes isoazotés suivants :

Tableau XV

Composition de régimes à II $p$. roo de protides.

\begin{tabular}{|c|c|c|c|c|c|c|}
\hline & $\mathrm{I} c$ & $\mathbf{I} b$ & $\mathrm{II} c$ & $\mathrm{II} b$ & $\mathrm{III}_{c}$ & $\mathrm{III} b$ \\
\hline Soia délinidé en $\mathrm{g}$ & & & & & & \\
\hline $\begin{array}{l}\text { Soja délipidé......... } \\
\text { Gluten de maïs ...... }\end{array}$ & $\underline{25}$ & 25 & $\overline{20}$ & $\overline{20}$ & $\overline{20}$ & $\overline{20}$ \\
\hline Sucre ............. & 60 & 60 & 65 & 65 & - & - \\
\hline Amidon de maîs $\ldots$ & - & - & - & - & 65 & 65 \\
\hline Margarine ........... & 5 & 5 & 5 & 5 & 5 & 5 \\
\hline Huile d'arachide.... & 3 & 3 & 3 & 3 & 3 & 3 \\
\hline Cellulose............ & 2 & 2 & 2 & 2 & 2 & 2 \\
\hline Mélange salin................. & 4 & 4 & 4 & 4 & 4 & 4 \\
\hline Mélange vitaminique sans $B$ I $2(\mathrm{r})$ & I & I & I & I & I & I \\
\hline Vitamine $B$ I 2. & 0 & + & o & + & o & + \\
\hline
\end{tabular}

Dans les séries b, la vitamine BI2 est ajoutée à raison de $0,03 \mathrm{mg}$ par $\mathrm{kg} \mathrm{sec}$ de régime.

Les observations portent sur la croissance, l'indice de consommation

(I) Composition décrite à la page 44 . 
et la réserve hépatique de BI2. Voici les résultats d'une expérience de quatre semaines :

Tableau XVI

Croissance des rats (40 $\mathrm{g}$ au départ).

\begin{tabular}{|c|c|c|c|}
\hline & Lots & Gains de poids (g) & Indices de consom- \\
\hline Ic & soja + sucre $\ldots \ldots \ldots \ldots \ldots \ldots$ & 20 & 7 \\
\hline $\mathrm{Ib}$ & soja + sucre $+B_{12} \ldots \ldots \ldots \ldots \ldots \ldots \ldots$ & 33 & 5,4 \\
\hline$I_{\mathrm{C}}$ & gluten + sucre $\ldots \ldots \ldots \ldots \ldots \ldots \ldots \ldots \ldots \ldots \ldots \ldots$ & $\begin{array}{r}3,3 \\
5\end{array}$ & 21,8 \\
\hline IIb & gluten + sucre $+\mathrm{B}_{\mathbf{2} 2} \ldots \ldots \ldots \ldots \ldots$ & 8 & I9 \\
\hline IIIc & gluten + amidon $\ldots \ldots \ldots \ldots \ldots \ldots \ldots$ & $I_{3}$ & 20 \\
\hline IIIb & gluten + amidon $+\mathrm{B}_{12} \ldots$ & $\ddot{9}$ & 23 \\
\hline
\end{tabular}

Au taux protidique de II p. Ioo, le soja permet une croissance moyenne de $0,7 \mathrm{I} g$ par jour ; le gluten de maïs couvre l'entretien, car on ne peut appeler croissance un développement aussi faible.

Il n'est pas surprenant qu'à taux azoté égal, les résultats soient considérablement meilleurs avec les protides du soja qu'avec le gluten de maïs : dans la hiérarchie chimique établie par Mitcheli (300 - zoI $302-303)$ les premiers sont dotés du coefficient 50, le second du coefficient 28. Le même écart s'observe dans les Valeurs Biologiques déterminées sur animal.

Mais le résultat le plus frappant est la diversité d'action de Br2. Avec le soja, elle améliore considérablement la croissance (bénéfice de 60 p. Ioo) et significativement l'indice de consommation. Avec le gluten de maïs, elle est inopérante sur le développement : avec ou sans B I2, les animaux grandissent mal et l'indice de consommation demeure extraordinairement élevé.

Le tableau suivant donne le taux des réserves hépatiques de Bız exprimé en $\gamma$.

\section{TABLEAU XVII}

Moyennes de 8 animaux par lot.

\begin{tabular}{|c|c|c|c|c|c|}
\hline & Lots & $\begin{array}{l}\text { Poids frais du } \\
\text { foie }(g)\end{array}$ & $\begin{array}{l}\text { Teneur en } \\
\text { B r } 2 \text { g de foie } \\
\text { frias (y) }\end{array}$ & $\begin{array}{l}\text { Teneur en B I } 2 \\
\text { du foie total ( } y)\end{array}$ & $\begin{array}{c}\text { B I } 2 \text { hépatique } \\
\text { par roog poids } \\
\text { vif }(y)\end{array}$ \\
\hline $\mathrm{Ic}$ & soja + sucre $\ldots \ldots$ & 4,6 & 0,05 & $0,2.3$ & 0,26 \\
\hline Ib & soja + sucre +13 r 2 & 3,7 & 0,19 & 0,60 & 0,38 \\
\hline IIc & gluten + sucre $\ldots \ldots \ldots$ & 3,4 & 0,06 & 0,19 & 0,39 \\
\hline IIb & gluten + sucre + 3 J $2 \ldots$ & 2,5 & 0,20 & $0,5 \mathrm{I}$ & 0,54 \\
\hline IIIc & grluten + amidon $\ldots . .$. & 3,6 & $0,0)$ & 0,32 & 0,48 \\
\hline IIIb & gluten + amidon $+B$ I 2 & 2,6 & 0,30 & 0,74 & 1,34 \\
\hline
\end{tabular}

Les réserves hépatiques sont entièrement fonction de l'apport de Bı2. si cette vitamine figure en nature dans le régime (séries b), le taux 
de BI2 hépatique est toujours élevé. En l'absence de BI2 alimentaire, ce taux demeure bas. I es teneurs hépatiques en Br 2 sont donc strictement indépendantes de 1'intensité du développement, puisque ce sont les rats alimentés au soja qui accusent la meilleure croissance, tout en ayant, par unité de poids vif, les plus faibles réserves hépatiques (colonne 4).

L'influence des glucides est particulièrement visible avec les lots III nourris d'amidon. Les rats de cette série présentent des réserves de BI2 nettement plus élevées que les animaux nourris au sucre (lots I et II) ; cependant, ici encore, la croissance est très mauvaise. Qu'il s'agisse d'apport alimentaire ou de réfections intestinales, l'absence de relations entre vitesse du développement et taux des réserves, interdit de considérer Br2 comme un facteur de croissance au sens général du terme.

Cependant, cette vitamine agit comme telle lorsqu'elle supplémente une ration à base de soja.

Il s'agit ici d'un cas particulier qui relève de la composition protéique de cette légumineuse. On l'avait déjà maintes fois signalé (66 - 285 328 - 330 - 429). Dès I948, RuBin et BIRD (362) montraient que les excréments de vache (riches en vitamine BI2) rehaussaient 1'efficacité nutritive du tourteau de soja. De même le soja cru, mauvais aliment de croissance du fait de l'indisponibilité de la méthionine qu'il renferme, est valorisé par cette vitamine B (I6). On avait également mentionné que la vitamine BIz élevait la Valeur Biologique de la caséine (Kon et col. (2I4)). Parallèlement, elle diminue les concentrations du sang en aminoacides et en azote protéique (223), ce qui implique une protéinogenèse accrue au niveau des tissus $(96-294)$ et du foie notamment $(366-367)$. Or, caséine et soja ont un point commun à savoir le facteur limitant qui est la méthionine. Dès lors, on conçoit très bien l'action favorable qu'exerce BI2 sur la croissance. Cette vitamine joue un rôle essentiel dans le transfert des groupements méthyle nécessaires à la synthèse de la méthionine ( 368 - 409). C'est parce qu'elle contrôle la transméthylation qu'elle possède une action d'épargne vis-à-vis de l'acide aminé soufré.

In vitro, DU VIGNEAUD a bien établi la synthèse possible de méthionine à partir d'homocystéine $(434-435-436)$. D'autres auteurs arrivent aux mêmes conclusions (38-39). L'addition de BI2 à des homogénéisats de foie provoque une réduction accrue des groupements $S-S$ en groupements $\mathrm{S}-\mathrm{H}$, sous l'effet de certains donateurs d'hydrogène. I'effet est encore plus marqué, si les foies proviennent d'animaux carencés en BI2 (I37 - 346). Des coupes de foies de rats carencés en BI2 synthétisent bien moins de méthionine à partir d'un mélange "choline (ou bétaïne)homocystéine " que les foies d'animaux normaux (325). On admet que les groupements $\mathrm{CH}_{3}$ peuvent être synthétisés par la flore microbienne à partir de corps divers : formiates, méthanol, sérine, glycine ( Io - 38 - I54 - 266-4II - 436). Cette réaction est commandée par la vitamine BI2. 
Le carbone $\alpha$ de la glycine se retrouve en plus grande quantité dans la méthionine que dans la sérine $(4 \mathrm{IO}-4 \mathrm{I} 2)$. On peut en tirer trois conclusions :

- le rôle de $\mathrm{Br} 2$ dans le métabolisme de divers acides aminés, glycine, sérine et cystéine, ne serait que partiel et secondaire $(\mathrm{I}-223-284)$; - la sérine ne serait pas un intermédiaire normal dans la transméthylation des formiates, puisque la réaction est beaucoup plus rapide en présence de glycine;

- la transméthylation se fait beaucoup plus aisément dans le cas de la méthionine que pour les autres composés (choline, bétaïne, etc.). Cependant, si la vitamine $\mathrm{BI} 2$ conditionne la synthèse de la méthionine, elle n'est cependant pas responsable de toutes les opérations de transméthylation (79). On sait qu'elle intervient notamment comme coenzyme de la xanthine-oxydase et de la bétaïne-homocystéine-transméthylase (458).

Ainsi ces faits renforcent nos conclusions.

I a vitamine $\mathrm{BI} 2$ peut donc apparaître comme un substitut alimentaire de la méthionine et, à ce titre, peut valoriser les régimes qui en sont déficients. Mais cette vicariance est limitée au métabolisme des aminoacides soufrés et ne saurait s'exercer pour corriger les défauts d'autres aminoacides. L'efficacité pour la croissance du gluten de maïs ne peut être améliorée par BI2 puisque cette protéine a la lysine comme facteur limitant.

Cette conclusion est celle d'autres expérimentateurs. L'addition de BI2 au maïs ne détermine ni augmentation de croissance, ni amélioration de l'indice de consommation. De même, cette vitamine ne permet pas 1a survie de jeunes rats nés de mères carencées en BIz et sevrés avec un régime strictement végétal.

En résumé, dans des essais poursuivis sur Rat blanc, la vitamine $\mathrm{BI} 2$ se montre capable d'augmenter l'efficacité de croissance d'un régime à base de soja, alors qu'elle est absolument inopérante à valoriser un régime à base de gluten de maïs. Cette observation trouve son explication dans la nature du facteur limitant : méthionine dans le premier cas, lysine dans le second.

Par ailleurs, on ne trouve aucune relation entre l'intensité du développement et le taux des réserves hépatiques de BI2.

I a vitamine BI2 n'est donc pas un facteur de croissance au sens complet du terme et son action favorable en présence de soja relève d'un processus métabolique très particulier : la transméthylation.

Transposées dans la pratique et sous réserve que les animaux de ferme réagissent comme le Rat, ces notions conduisent à la conclusion suivante : l'usage de $\mathrm{BI} 2$ pour stimuler le développement et améliorer l'indice de consommation ne doit pas être généralisé à tous les types de 
rations. Il n'aura de signification et ne donnera les résultats escomptés qu'avec des régimes déficients en aminoacides soufrés. Or, la majorité des céréales, sinon toutes, ont la lysine comme facteur limitant et ne peuvent dès lors être valorisées par BI2. L'intérêt incontestable de cette vitamine réside dans ses propriétés hématopoiétiques. Elles peuvent à elles seules rendre compte de son importance pour l'éclosabilité de l'œuf, sans qu'il soit besoin de faire appel à un rôle de facteur de croissance beaucoup plus aléatoire.

\section{III. - CONCLUSIONS GÉNÉRALES}

On voit ainsi la complète dissociation des rôles de la vitamine BIz et de l'auréomycine. La stimulation de la croissance par cette dernière n'implique nullement un relai par la vitamine BI2 et relève d'un processus métabolique direct dont nous allons tenter de donner un essai d'interprétation.

\section{CHAPITRE, IV}

\section{SIGNIFICATION DE L'INFLUENCE DE L'A UREOMYCINE SUR LA CROISSANCE D'APRES L'ÉTUDE DES BILANS}

PAR

\section{CALET et A. RERAT (1)}

Le fait d'être arrivé à dissocier le rôle de la vitamine Br2 et de l'auréomycine n'explique nullement le mécanisme d'action selon lequel cette dernière substance se comporte comme facteur de croissance. Un des moyens d'aborder le problème consiste à déterminer les bilans nutritionnels d'animaux recevant ou non cet antibiotique. Il permet en outre de trancher le débat relatif à l'intervention des antibiotiques dans le métabolisme azoté.

Élargissant la notion de pouvoir d'épargne, primitivement réservée aux seules vitamines $B$, certains auteurs ont prétendu que les antibiotiques avaient un effet d'épargne pour les protéines chez le Porc (893) et chez les volailles $(44-\mathrm{I} 77-234-283-292-383-393-$ 396). CATron (92) a même proposé des normes protidiques plus basses en présence d'antibiotiques. Cette conception repose uniquement sur le fait que de jeunes animaux carencés en azote grandissent mieux s'ils reçoivent des antibiotiques.

(I) Avec la collaboration technique de $\mathrm{M} \mathrm{I}^{\mathrm{mes}}$ de Saintaurain et Macaire. 
A l'opposé, de très nombreux expérimentateurs travaillant sur volailles arrivent à la conclusion que, loin de réduire les besoins protidiques, les antibiotiques les augmentent et en particulier élèvent les besoins en acides aminés $(235-255-263-33 \mathrm{I}-394)$. D'où certaines pratiques qui consistent à surcharger les rations en protides pour obtenir la meilleure efficacité des antibiotiques.

\section{CONDITIONS EXPÉRIMENTALES}

I $\mathrm{e}$ régime de base est le même que précédemment, c'est-à-dire un régime à base de farine blanche supplémenté en lipides, en cellulose, en sels et enrichi en toutes les vitamines à dose optima. En ce qui concerne l'apport protidique, il est quantitativement trop faible (Io,5 p. Ioo de protides) et qualitativement médiocre (Valeur Biologique $=50$ ).

Des lots de 9 rats pesant $37 \mathrm{~g}$ an départ sont répartis dans les 4 séries suivantes :

- Lot I : $\mathrm{R} \mathrm{B}$

- Lot II : $\quad \mathrm{R} \mathrm{B}+\mathrm{BI2}(30 \% / \mathrm{kg})$

- Lot III : $\quad \mathrm{R} \mathrm{B}+$ Auréomycine (100 $\mathrm{mg} / \mathrm{kg}$ )

- Lot IV : $\quad \mathrm{R} \mathrm{B}+\mathrm{B.T} 2(30 \% \mathrm{~kg})+$ Auréomycine (I00 mg kg).

L'expérience dure 9 semaines au cours desquelles on établit les bilans complets d'azote (C.U.D. et C.R.). Puis on sacrifie les animaux, on prélève la peau totale et la masse musculaire de l'arrière train dont on détermine la teneur en eau.

\section{RÉSULTATS EXPÉRIMENTAUX}

Le tableau XVIII rapporte l'ensemble de nos résultats.

D'une manière générale, on peut dire que, pour chaque lot, les résultats sont très homogènes; on est frappé par la valeur des écarts-types qui sont très faibles (surtout pour les coefficients de digestibilité).

La teneur en eau des tissus est rigoureusement constante chez tous les animaux ; ce qui veut dire que l'effet de croissance de l'auréomycine ne porte pas sur une plus grande hydratation de la carcasse.

La digestibilité brute de la ration (matière sèche) est la même pour tous les lots ; ce qui veut dire que l'effet de croissance de l'auréomycine ne s'explique pas par une meilleure utilisation digestive des substances organiques.

De même le C. U.D. azoté est identique pour tous les lots. Ce résultat a été confirmé ultérieurement à notre propre observation (97- r62). Ces résultats vont à l'encontre d'une action spécifique des antibiotiques sur 1'absorption intestinale des nutriments azotés, notion qui avait été pro- 
TABLEAU XVIII

\begin{tabular}{|c|c|c|c|c|}
\hline Lots & $\underset{\mathrm{RB}}{\mathrm{I}}$ & $\mathrm{II}$ & $\begin{array}{l}\mathrm{III} \\
\mathrm{RB}+\mathrm{Au} \\
\text { réomycine }\end{array}$ & $\left\{\begin{array}{c}\mathrm{IV} \\
\mathrm{RB}+\mathrm{B} \mathbf{\text { I2 }} \\
+\begin{array}{c}\text { Auréo } \\
\text { mycine }\end{array}\end{array}\right.$ \\
\hline $\begin{array}{l}\mathrm{I} .- \text { Observalions in vivo } \\
\text { Gain de poids total }(\mathrm{g}) \ldots \ldots \ldots \ldots \ldots \\
\text { Indice de consommation } \ldots \ldots \ldots \ldots \ldots\end{array}$ & $\begin{array}{l}24,5 \\
14,4\end{array}$ & $\begin{array}{l}23,4 \\
14,5\end{array}$ & $\begin{array}{r}35,3 \\
9,9\end{array}$ & $\begin{array}{c}34,5 \\
9,9\end{array}$ \\
\hline 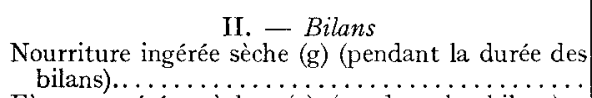 & $I \mp 4,2$ & $\mathrm{I} 2 \mathrm{I}, 4$ & I 28,6 & $1 \pm 4,9$ \\
\hline $\begin{array}{l}\text { Fèces excrétées sèches (g) (pendant les bilans).. } \\
\text { Coefficient de digestibilité brute............... }\end{array}$ & $\begin{array}{r}7,9 \\
93,0\end{array}$ & $\begin{array}{r}7,4 \\
93,8\end{array}$ & $\begin{array}{r}8,2 \\
8,6 \\
9,6\end{array}$ & $\begin{array}{r}7,9 \\
7,7 \\
93,3\end{array}$ \\
\hline Ecart-type de ce coefficient .................. & 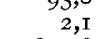 & $\begin{array}{r}93,0 \\
\mathbf{1}, 7\end{array}$ & $\begin{array}{r}9,4 \\
\mathrm{I}, 4\end{array}$ & $\begin{array}{l}93,3 \\
2,15\end{array}$ \\
\hline $\begin{array}{l}\text { Azote ingéré }(\mathrm{mg}) \ldots \ldots \ldots \ldots \ldots \ldots \ldots \\
\text { Azote fécal }(\mathrm{mg}) \ldots \ldots \ldots \ldots \ldots \ldots\end{array}$ & $\begin{array}{r}\text { I } 820,8 \\
28,0\end{array}$ & $\begin{array}{r}\text { I } 821,8 \\
27,0\end{array}$ & 2101,6 & I 936,8 \\
\hline $\begin{array}{l}\text { Azote fécal (mg) ..... } \\
\text { Azote absorbé (mg). }\end{array}$ & I 792 & г 794 & 2075 & $\begin{array}{l}23,0 \\
\text { I 9I4 }\end{array}$ \\
\hline C. U. D. azoté $\ldots \ldots \ldots \ldots$ & 98,4 & 98,4 & 98,8 & 98,8 \\
\hline Azote urinaire $(\mathrm{mg})$.............. & I 096,0 & I 080,0 & I 214,0 & I 170,8 \\
\hline Azote retenu $(\mathrm{mg}) \ldots \ldots \ldots \ldots \ldots$ & 696,8 & 712,0 & 734,4 & 743,2 \\
\hline Ecart-type de l'azote retenu ........ & 63,0 & 62,4 & 59,4 & 46,4 \\
\hline Coefficient de rétention & 38,8 & $39, \mathrm{I}$ & 38, & 38,8 \\
\hline Ecart-type du C. R. . . . . . . . . . & 7,32 & 8,10 & $8, \infty 0$ & 6,00 \\
\hline 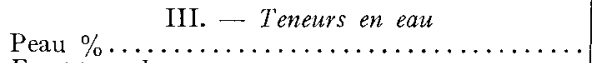 & $6 \mathrm{I}, 0$ & 60,1 & 62,5 & 60,6 \\
\hline 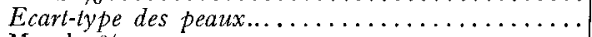 & 4,16 & 4,12 & 3,8 & 3,93 \\
\hline Muscle $\% \ldots \ldots \ldots$ & 72,8 & 73,0 & 72,3 & 72,2 \\
\hline Ecart-type des muscles & $\mathrm{r}, 9 \mathrm{I}$ & $2,3^{8}$ & 2,79 & $\mathbf{I}, 5^{1}$ \\
\hline
\end{tabular}

posée par. différents auteurs se basant, il est vrai, sur des considérations indirectes : perfusion de l'intestin isolé, pouvoir tensio-actif de certains antibiotiques, effets de ces substances sur l'épaisseur de la paroi intestinale (voir introduction).

Les coefficients de rétention azotée sont identiques pour tous les lots. L'auréomycine n'a pas d'action sur les quantités d'azote retenu, ce qui montre qu'elle n'améliore pas la Valeur Biologique de la farine blanche et n'exerce pas d'action d'épargne vis-à-vis des amino-acides. Le tableau XVIII montre de plus que la valeur absolue de l'azote retenu des différents lots reste inchangée. Ceci revient à dire que le bénéfice pondéral provoqué par l'antibiotique ne correspond nullement à une protéinogenèse accrue. On le démontre encore plus clairement en confrontant le résultat des pesées des animaux et les études de leur bilan. Les premières indiquent les variations pondérales brutes, alors que les secondes permettent d'apprécier la croissance active. La quantité de protides élaborés est obtenue en multipliant l'azote retenu par le coefficient de transformation de l'azote en protides. Le gain de tissu protéique est alors calculé à partir de la teneur en eau telle que nous l'avons mesurée soit : 73 p. Ioo.

Voici les résultats : 


\section{TABleau XIX}

Comparaison du gain de poids corporel et de la quantité de tissu protéique formé au cours des bilans

\begin{tabular}{|c|c|c|c|}
\hline Régime & Gain de poids (g) & $\begin{array}{c}\text { Tissu } \\
\text { protéique }(\mathrm{g})\end{array}$ & Différence \\
\hline 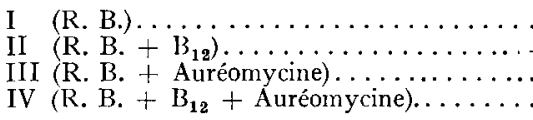 & $\begin{array}{l}16,5 \\
17,5 \\
25,2 \\
24,8\end{array}$ & $\begin{array}{l}16,2 \\
16,5 \\
\mathrm{I} 7,0 \\
\mathrm{r} 7, \mathrm{I}\end{array}$ & $\begin{array}{l}0,3 \\
1,0 \\
8,2 \\
7,6\end{array}$ \\
\hline
\end{tabular}

\section{Diseussion.}

Nous examinerons successivement le problème des antibiotiques et celui de la vitamine BI2.

$I^{\circ}$ Chez tous les animaux ne recevant pas d'auréomycine, la concordance est excellente entre le gain de poids vif et l'accroissement du tissu protéique. Nous sommes là devant une croissance active correspondant à la seule protéinogenèse. Par contre, chez tous les rats soumis à l'auréomycine il existe un indéterminé considérable dans l'analyse des variations pondérales. Il y a donc quelque chose qui s'ajoute à la croissance protéique puisque celle-ci demeure inchangée et ne peut nullement rendre compte de la variation de poids vif.

Si nous concluons à la non-intervention des antibiotiques comme facteurs de protéinogenèse, comme le montrent d'ailleurs certains résultats publiés ultérieurement à nos propres observations $(387-407)$, on ne saurait cependant leur dénier toute influence sur le métabolisme protéique. Mais cet effet semble porter plus sur le catabolisme que sur l'anabolisme et intéresser davantage la flore que l'organisme lui-même. Voici les faits :

- les antibiotiques ont une action variable avec la nature des protides alimentaires (soja, arachide, poisson) $(76-$ I22). La farine de poisson présente une efficacité protidique supérieure à celle de l'arachide ; cette différence n'apparaît plus en présence d'auréomycine. On a pensé que l'antibiotique débarrasse l'intestin d'une flore engendrée par l'arachide et non par le poisson, flore prédatrice d'aminoacides.

A l'appui de cette idée, Françors vient d'apporter d'utiles précisions sur le rôle des antibiotiques dans le métabolisme azoté de la flore intestinale (I7I - 297):

- in vitro, cultivée sur substrat complexe ou sur aminoacides purs, la flore intestinale du Porc donne normalement naissance à de l'ammoniac. ce qui implique une désamination préalable; les antibiotiques contrarient cette réaction et inhibent la formation d'ammoniac; 
- in vivo, il existe une corrélation entre l'indice de croissance ${ }^{1}$ ) des antibiotiques et leur aptitude à inhiber la désamination mesurée par la teneur en ammoniac du côlon et du cæcum.

Autrement dit, l'antibiotique garantirait les aminoacides contre la désamination bactérienne, ce qui aurait pour effet une grande mise à la disposition de l'hôte de formes aminées efficaces. On pourrait ainsi expliquer l'effet d'épargne signalé par quelques auteurs.

Les résultats de BRANion et Hili $\left(5^{8}\right)$ vont à l'appui de la théorie : l'ingestion de poisson décomposé, normalement générateur de putréfactions intestinales, n'a aucun effet toxique en présence d'antibiotiques.

On comprend mal dès lors, les affirmations de JonEs et Combs d'une part (236), qui signalent l'inaptitude de l'auréomycine à valoriser l'efficacité pour la croissance et l'indice de consommation de régimes contenant des sels d'ammonium et d'autre part, de divers auteurs (44-332-394423) qui enregistrent une élévation des besoins en certains aminoacides, lysine, thréonine, tryptophane, en présence d'antibiotique.

Nos travaux ne nous permettent pas d'intervenir dans le débat, mais d'affirmer seulement que, dans nos essais, l'augmentation de poids due à 1'auréomycine ne s'inscrit pas au niveau de la rétention azotée. Cette conclusion est conforme à 1'avis de nombreux auteurs $(54-166-224-$ $332-373-448)$.

BERRY et Schuck ont même écrit que les antibiotiques diminuaient la rétention azotée (42). Les opinions contraires existent mais elles sont rares $(3-77-296-45 \mathrm{I})$.

Quant à la constance d'hydratation tissulaire des animaux recevant ou non des antibiotiques, elle est généralement admise avec naturellement quelques opinions contraires. BRAUDE et JOHNSON (62) observent même que les antibiotiques auraient tendance à déshydrater les tissus : ceux-ci font boire plus, mais uriner encore davantage. Ces auteurs ont déduit de ces faits une théorie métabolique que nous examinerons par la suite.

$2^{\circ}$ La vitamine $\mathrm{B} \mathbf{2} 2$ est inopérante à modifier la valeur du bilan azoté, comme elle est inopérante à valoriser l'efficacité pour la croissance du régime. D'autres auteurs $(9-48-98-\mathrm{I} 88-249-272)$ étaient arrivés aux mêmes conclusions, tant pour le bilan que pour la restauration du poids et la régénération des protides hépatiques (433). Ainsi cette vitamine n'a qu'un rôle très restreint parmi les facteurs accessoires qui conditionnent l'efficacité protidique.

(1) Grâce à l'étude statistique de très nombreux résultats, on a pu établir pour chaque antibiotique un indice de croissance qui est la valeur moyenne du taux de croissance dû à l'antibiotique calculée en p. roo du régime de base. 


\section{Conclusions.}

Arrivés à ce stade, nous pouvons donc mieux délimiter le mode d'action de l'auréomycine sur la croissance en éliminant plusieurs hypothèses.

L'efficacité pour la croissance de cet antibiotique ne s'explique :

- ni par son interchangeabilité avec la vitamine Br2, qui d'ailleurs ne valorise pas indistinctement toutes les protéines végétales,

- ni par une meilleure utilisation digestive de la ration,

- ni par une hydratation plus élevée des tissus,

- ni par une protéinogenèse accrue.

Elle semble donc relever uniquement de 1'augmentation soit de la lipogenèse, soit des éléments minéraux du squelette.

C'est cette preuve que nous rechercherons dans l'analyse des carcasses. 